\title{
Young, active radio stars in the AB Doradus moving group
}

\author{
R. Azulay ${ }^{1,2, \star}$, J. C. Guirado ${ }^{3,1}$, J. M. Marcaide ${ }^{1}$, I. Martí-Vidal ${ }^{4}$, E. Ros ${ }^{2,1,3}$, E. Tognelli5 ${ }^{5,6}$, \\ F. Hormuth ${ }^{7}$, and J. L. Ortiz ${ }^{8}$
}

\author{
1 Departament d'Astronomia i Astrofísica, Universitat de València, C. Dr. Moliner 50, 46100 Burjassot, València, Spain \\ e-mail: Rebecca.Azulay@uv.es \\ 2 Max-Planck-Institut für Radioastronomie, Auf dem Hügel 69, 53121 Bonn, Germany \\ 3 Observatori Astronòmic, Universitat de València, Parc Científic, C. Catedrático José Beltrán 2, 46980 Paterna, València, Spain \\ 4 Onsala Space Observatory, Chalmers University of Technology, 43992 Onsala, Sweden \\ 5 Department of Physics "E.Fermi”, University of Pisa, Largo Bruno Pontecorvo 3, 56127 Pisa, Italy \\ 6 INFN, Section of Pisa, Largo Bruno Pontecorvo 3, 56127 Pisa, Italy \\ 7 Max-Planck-Institut für Astronomie, Koenigstuhl 17, 69117 Heidelberg, Germany \\ 8 Instituto de Astrofísica de Andalucía (IAA-CSIC), Apt 3004, 1808 Granada, Spain
}

Received 14 October 2016 / Accepted 14 March 2017

\begin{abstract}
Context. Precise determination of stellar masses is necessary to test the validity of pre-main-sequence (PMS) stellar evolutionary models, whose predictions are in disagreement with measurements for masses below $1.2 M_{\odot}$. To improve such a test, and based on our previous studies, we selected the AB Doradus moving group (AB Dor-MG) as the best-suited association on which to apply radio-based high-precision astrometric techniques to study binary systems.

Aims. We seek to determine precise estimates of the masses of a set of stars belonging to the AB Dor-MG using radio and infrared observations.

Methods. We observed in phase-reference mode with the Very Large Array (VLA) at $5 \mathrm{GHz}$ and with the European VLBI Network $($ EVN) at $8.4 \mathrm{GHz}$ the stars HD 160934, EK Dra, PW And, and LO Peg. We also observed some of these stars with the near-infrared CCD AstraLux camera at the Calar Alto observatory to complement the radio observations.

Results. We determine model-independent dynamical masses of both components of the star HD 160934, A and c, which are $0.70 \pm 0.07 M_{\odot}$ and $0.45 \pm 0.04 M_{\odot}$, respectively. We revised the orbital parameters of EK Dra and we determine a sum of the masses of the system of $1.38 \pm 0.08 M_{\odot}$. We also explored the binarity of the stars LO Peg and PW And.

Conclusions. We found observational evidence that PMS evolutionary models underpredict the mass of PMS stars by $10 \%-40 \%$, as previously reported by other authors. We also inferred that the origin of the radio emission must be similar in all observed stars, that is, extreme magnetic activity of the stellar corona that triggers gyrosynchrotron emission from non-thermal, accelerated electrons.
\end{abstract}

Key words. binaries: general - stars: pre-main sequence - radio continuum: general - astrometry

\section{Introduction}

Stellar evolution models are an essential tool to infer star fundamental parameters such as radius, mass, and/or age from luminosity/temperature-based relationships (e.g., Baraffe et al. 1998; Chabrier et al. 2000). The reliability of the models has long been tested and validated by the overall good agreement between the predictions of stellar models and measurements. However, only recently, accurate measurements of stellar masses and radii have become accessible, especially in the case of low- and very low-mass stars, thus allowing more stringent tests on stellar models. In the particular case of pre-main-sequence (PMS) stars the models show an increasing difficulty in accurately reproducing some of the characteristics of star with masses below $1.2 M_{\odot}$ (e.g., Hillenbrand \& White 2004).

Therefore, the calibration of the evolutionary models of lowmass PMS stars is an important and challenging task, since it requires precise and independent measurements of luminosities and masses to be compared with theoretical predictions. Several authors have highlighted these facts in previous works but, nevertheless, there is not enough observational data that can help

\footnotetext{
* Guest student of the International Max Planck Research School for Astronomy and Astrophysics at the Universities of Bonn and Cologne.
}

to improve the models yet (Hillenbrand \& White 2004; Stassun et al. 2004; Mathieu et al. 2007; Gennaro et al. 2012).

The study of binary stars belonging to young moving groups, whose main feature is the common age of their members, is a reasonable approach to increase the number of PMS stars with dynamically determined masses. Several of these moving groups have recently been discovered (Zuckerman \& Song 2004; Torres et al. 2008). Among all of these groups, the AB Doradus moving group (AB Dor-MG) is the most suitable to carry out the study; because this group is the closest moving group, the estimated age is relatively accurate and contains stars with significant emission at radio wavelengths (Guirado et al. 2006, 2011; Jason et al. 2007; Azulay et al. 2014, 2015). This last feature is essential because it allows us to use radio interferometry techniques to obtain astrometric information. Using these techniques it is possible to achieve angular resolutions in the sub-milliarcsecond (sub-mas) range, which are needed to solve and study in detail the kinematics (proper motion, parallax, and possible orbits) of the stellar systems.

In this context, we have made several contributions to stars belonging to the AB Dor-MG, namely, AB Dor A/C (Guirado et al. 2006, 2011), AB Dor Ba/Bb (Azulay et al. 2015), and HD 160934 (Azulay et al. 2014). In the two first cases, a 
VLBI-driven astrometric study resulted in the precise estimate of the dynamical mass of the individual components, providing relevant results in terms of calibration of the mass-luminosity relationship for young, low-mass objects. Regarding the binary HD 160934, we reported the discovery of compact radio emission from both components of the system, which opened the possibility to a further astrometric monitoring of its orbital motion.

Given the remarkable scientific output of $\mathrm{AB}$ Dor $\mathrm{A} / \mathrm{C}$, $\mathrm{AB}$ Dor $\mathrm{Ba} / \mathrm{Bb}$, and $\mathrm{HD} 160934$, we considered it appropriate to include new similar stars, that is, young binaries that are luminous both in infrared and radio wavelengths. In fact, other stars in the AB Dor-MG are fast rotators, showing traces of magnetic activity (as stellar spots) that well could be radio emitters. The previous reasoning was the main motivation to initiate a study of the radio emission of AB Dor-MG members beyond the systems already studied.

In this paper we present the results of a VLA/VLBI radio study of PMS stars members of the AB Dor-MG, namely, HD 160934, EK Dra, PW And, and LO Peg. In particular, we focus on the VLBI observations of HD 160934, from which we were able to monitor astrometrically the relative orbit (of the component HD $160934 \mathrm{~A}$ respect to the component HD $160934 \mathrm{c}$ ) and the absolute orbit (reflex motion of the component HD $160934 \mathrm{~A}$ with respect to a external quasar) and, thereby, to determine dynamical individual masses of both components of the star, which enabled further comparisons with stellar models. We also report on VLBI observations of the other three stars addressed to determine their fundamental parameters (EK Dra) or explore its possible binarity (PW And and LO Peg).

\section{Observations and data reduction}

\subsection{VLA observations}

We analyzed archival VLA data ${ }^{1}$ of the stars EK Dra, PW And, and LOPeg observed at $8.4 \mathrm{GHz}$ in $\mathrm{AB}$ (EKDra) and CD (PW And and LO Peg) configurations on 1993 January 29, 1993 September 16, and 1996 May 5, respectively (see Table 1). In all cases, the effective bandwidth was $50 \mathrm{MHz}$ and both right- and left-handed circular polarizations were collected. For EK Dra the observation lasted $10.5 \mathrm{~h}$, the source $3 \mathrm{C} 48$ was used as the primary flux calibrator and the source $1435+638$ was selected as the phase calibrator. For PW And, the observation lasted $10 \mathrm{~h}$, and the flux and phase calibrators were $0137+331$ and $0029+349$, respectively. For LO Peg, the observation lasted $5.5 \mathrm{~h}$, the source $0137+331$ was used as primary flux calibrator, and the source $2115+295$ was selected as phase calibrator. VLA observations of HD 160934 are described in Azulay et al. (2014) and included in Table 1 for completeness.

To reduce all three experiments, we used standard routines of the Astronomical Image Processing System (AIPS, 31DEC15 version) program of the National Radio Astronomy Observatory (NRAO), which we summarize in turn. We flagged data, both obvious outliers or data segments selected after careful checking of the observing logs, which constituted a small fraction of the complete data set; we determined the flux density of the primary calibrator, we calculated the flux density of the phase calibrator from the primary flux calibrator, and we used the solutions derived from the calibrators to calibrate the amplitudes and phases of the target through linear interpolation. These calibrated data were imported to the DIFMAP software package

\footnotetext{
1 Projects AG0377, ADA000, and ABO691 available at the VLA data archive https://archive.nrao.edu/archive/advquery.jsp
}

(Shepherd 1994) to obtain the images of the stars. These resulting images are shown in Figs. 10, 15, and 18 and are discussed in the next section.

\subsection{VLBI observations}

The previous VLA observations certified the presence of radio emission on HD 160934, EK Dra, PW And, and LO Peg; to study their compact structures, we carried out VLBI observations at $5 \mathrm{GHz}$ between 2012 and 2014 with the EVN (see Table 1). Results of the first VLBI epoch on HD 160934 were already presented in Azulay et al. (2014) but they have been reanalyzed here in the context of new observations. For each experiment we observed an overall time of $10 \mathrm{~h}$ and both polarizations were recorded with a rate of $1024 \mathrm{Mbps}$ (two polarizations, eight subbands per polarization, $16 \mathrm{MHz}$ per sub-band, and two bits per sample). After each observation, the data were correlated with the EVN MkIV data processor at the Joint Institute for VLBI in Europe (JIVE), Dwingeloo, The Netherlands.

As we are studying weak sources, we used the phasereferencing technique to facilitate its detection; for that, we interleave scans of the target sources with ICRF quasars. The quasars selected were J1746+6226, J1441+6318, J0015+3216, and J2125+2442 for HD 160934, EK Dra, PW And, and LO Peg, respectively (separated by $1.50^{\circ}, 1.04^{\circ}, 1.48^{\circ}$, and $1.87^{\circ}$, respectively). The cycles target-calibrator-target lasted about six minutes in all cases.

We reduced each experiment using AIPS in a standard procedure briefly described here. The initial reduction included amplitude calibration using system temperatures and antenna gains, corrections of parallactic angle, and ionosphere. We applied fringe fitting on the calibrator to determine phase offsets and applied the solutions to the target source. Later, we imported the resulting data to DIFMAP to obtain uniformly weighted maps of the calibrator (Fig. 1, 9, 16, and 19). We obtained the image through a process of self-calibration iterations of the amplitude and phase with deconvolutions using the CLEAN algorithm, which allowed us to determine both the amplitude scaling corrections and self-calibrated phase for each antenna. Back to AIPS, we applied these corrections to the target source to obtain the phase-referenced naturally weighted images (Figs. 2, 11, and 17). We analyze the details in the next section.

\subsection{AstraLux observations}

HD 160934 and EKDra were also observed with the Lucky Imaging AstraLux camera (Hormuth et al. 2008) at the Calar Alto $2.2 \mathrm{~m}$ telescope. The Lucky Imaging technique permits the reducuction of distortions due to atmosphere by acquiring a large number of short-exposure images and combining the best few percent of high-quality images to obtain a final image that is relatively unaffected by atmospheric turbulence (Hormuth et al. 2007, and reference therein). The observations of HD 160934 were carried out on 2013 June 24 and 2015 November 19; the observations of EK Dra were carried out on 2013 June 24 2013. In all cases, we used two different filters, $\operatorname{SDSS} i^{\prime}$ and $\operatorname{SDSS} z^{\prime}$, and we took 10000 individual frames with exposure times of $30 \mathrm{~ms}$ each. The individual frames were dark and flat corrected before selecting the best $10 \%$ of the accquisitions. The final images were constructed by filtering and resampling the selected frames, and then combining them with the Drizzle algorithm (Fruchter \& Hook 2002). We also observed the stars at the center of the globular cluster M15, whose positions 
Table 1. Journal of observations.

\begin{tabular}{|c|c|c|c|c|c|}
\hline Source & Date (Epoch) & VLA configuration & UT Range & $\begin{array}{l}\text { Beam size } \\
\text { [arcsec] }\end{array}$ & $\begin{array}{l}\text { PA } \\
{\left[{ }^{\circ}\right]}\end{array}$ \\
\hline HD 160934 & 13 Feb. 2009 & B & $17: 15-22: 15$ & $1.01 \times 0.79$ & -35.9 \\
\hline EK Dra & 29 Jan. 1993 & $\mathrm{AB}$ & $01: 25-12: 00$ & $0.74 \times 0.33$ & -72.8 \\
\hline PW And & 16 Sep. 1993 & $\mathrm{CD}$ & 06:00-16:00 & $10.90 \times 3.28$ & 80.5 \\
\hline LOPeg & 5 May 1996 & $\mathrm{CD}$ & 12:50-18:10 & $7.63 \times 3.44$ & 82.0 \\
\hline Source & Date (Epoch) & VLBI array ${ }^{a}$ & UT Range & $\begin{array}{l}\text { Beam size } \\
\text { [mas] }\end{array}$ & $\begin{array}{l}\mathrm{PA} \\
{\left[{ }^{\circ}\right]}\end{array}$ \\
\hline HD 160934 & 30 Oct. 2012 & Ef, Wb, Jb, On, Mc, Nt, Tr, Ys, Sv, Zc, Bd, Ur, Sh & 10:30-20:30 & $2.29 \times 1.59$ & -28.4 \\
\hline " & 23 May 2013 & & 21:00-07:00 & $2.20 \times 1.76$ & -31.3 \\
\hline " & 5 Mar. 2014 & " & $02: 00-12: 00$ & $2.05 \times 1.59$ & -27.3 \\
\hline EK Dra & 29 Oct. 2012 & Ef, Wb, Jb, On, Mc, Nt, Tr, Ys, Sv, Zc, Bd, Ur, Sh & $07: 30-17: 30$ & $2.19 \times 1.71$ & -34.1 \\
\hline " & 27 May 2013 & & $17: 45-03: 45$ & $1.30 \times 1.02$ & -30.5 \\
\hline " & 5 Mar. 2014 & " & $16: 30-02: 30$ & $1.79 \times 0.96$ & -13.0 \\
\hline Pw And & 26 Oct. 2014 & Ef, Wb, Jb, On, Nt, Tr, Ys, Sv, Zc, Bd, Sh & $16: 30-02: 30$ & $4.63 \times 2.84$ & 0.4 \\
\hline LOPeg & 23 Oct. 2014 & Ef, Wb, Jb, On, Nt, Tr, Ys, Sv, Zc, Bd, Sh, Hh & 13:00-23:00 & $1.21 \times 0.89$ & -84.4 \\
\hline
\end{tabular}

Notes. ${ }^{(a)}$ Ef: Effelsberg, Wb: Westerbork, Jb: Jodrell Bank, On: Onsala, Mc: Medicina, Nt: Noto, Tr: Torun, Ys: Yebes, Sv: Svetloe, Zc: Zelenchukskaya, Bd: Badary, Ur: Urumqi, Sh: Shanghai, Hh: Hartebeesthoek.

were used for astrometric calibration in the way described in Hormuth et al. (2007). At each observation, the field of view was $24^{\prime \prime} \times 24^{\prime \prime}$ in a $512 \times 512$ pixel frame. The resulting images are shown in Figs. 3 and 12. Details are shown in the next section.

\section{Discussion on individual sources}

\section{1. $H D 160934$}

HD 160934 (=HIP 86346) is a young, very active, binary star (Zuckerman et al. 2004; López-Santiago et al. 2006). The two components, HD 160934 A and HD 160934 c, were first reported by Galvez et al. 2006). This star has spectral type K7Ve (Schlieder et al. 2012) and it is placed at a distance of $\sim 33 \mathrm{pc}$ (van Leeuwen 2007).

Several studies of HD 160934 have been carried out so far with different techniques. Radial velocity measurements were performed by Henry et al. (1995), Zuckerman et al. (2004), Gálvez et al. (2006), Griffin \& Filiz Ak (2010), and Griffin (2013). Relative astrometry through infrared imaging was reported by Hormuth et al. (2007) and Lafrenière et al. (2007). Moreover, relative astrometry from aperture-masking interferometry was provided by Evans et al. (2012). In Azulay et al. (2014), we reported the discovery of the radio emission of both components of the pair. There, we used that discovery to carry out relative astrometry and to determine new orbital elements and mass estimates.

Regarding our new VLBI images, only a single component (assigned to A) is detected in 2013.392 (the upper bound to the radio emission of the component $\mathrm{c}$ is $0.01 \mathrm{mJy}$ ). In contrast, in the images corresponding to 2014.175, and as it happened in the observations of 2012.830 reported in Azulay et al. (2014), two point-like features are clearly seen. Those two point-like features can readily be associated with components $\mathrm{A}$ and $\mathrm{c}$ of the binary HD 160934. Circular Gaussian least-squares-fitted parameters for the two components are listed in Table 2.

In the AstraLux images, meanwhile, the two components are not distinguished in the 2013 images whereas they are in those of 2015 , where $\mathrm{c}$ is near the apoastron. We fitted a binary model to
Table 2. Circular Gaussian estimates of the components of HD 160934.

\begin{tabular}{cccc}
\hline \hline Epoch & Component & $\begin{array}{c}\text { Flux } \\
(\mathrm{mJy})\end{array}$ & $\begin{array}{c}\text { Diameter } \\
(\mathrm{mas})\end{array}$ \\
\hline 2012.830 & $\mathrm{~A}$ & $0.16 \pm 0.01$ & $2.76 \pm 0.17$ \\
& $\mathrm{c}$ & $0.06 \pm 0.01$ & $1.20 \pm 0.20$ \\
\hline 2013.392 & $\mathrm{~A}$ & $0.05 \pm 0.01$ & $2.52 \pm 0.46$ \\
\hline 2014.175 & $\mathrm{~A}$ & $0.13 \pm 0.01$ & $2.76 \pm 0.20$ \\
& $\mathrm{c}$ & $0.06 \pm 0.01$ & $1.83 \pm 0.30$ \\
\hline
\end{tabular}

find the separation and flux ratio of the latter images; the results are shown in Table 3, along with previous estimates.

\subsubsection{Orbital parameters}

In order to carry out an astrometric study, we measured the relative position of the pair A-c directly on the maps shown in Fig. 2 (except for epoch 2013.39); we also measured the absolute position of the main component A, whose coordinates in Fig. 2 are in turn referenced to the position of the external quasar (Table 4). We augmented our data set with the relative position in Fig. 3 of 2015 and with previous orbital measurements reported by Evans et al. (2012), Hormuth et al. (2007), and Lafrenière et al. (2007). Table 4 shows all the archive positions available for the system.

We estimated the Keplerian parameters of HD 160934 via a weighted least-squares fit that combined the absolute positions of component $\mathrm{A}$ and all the relative positions constructed as $\mathrm{A}-\mathrm{c}$, that is, taking $\mathrm{c}$ as reference. We followed a similar approach to that used in Azulay et al. (2015) for another star of the AB Dor-MG, that is, AB Dor B, solving simultaneously for the absolute and relative orbits using the Thiele-Innes elements and the Levenberg-Marquardt algorithm. In practice, we proceed with two steps:

1. We obtained a priori values of the orbital elements from a previous least-squares fit to the, more numerous, differential data; in particular we estimated values for the period $P$ $(10.33 \mathrm{yr})$, semimajor axis of the relative orbit $a_{\mathrm{rel}}\left(0^{\prime \prime} .152\right)$, 

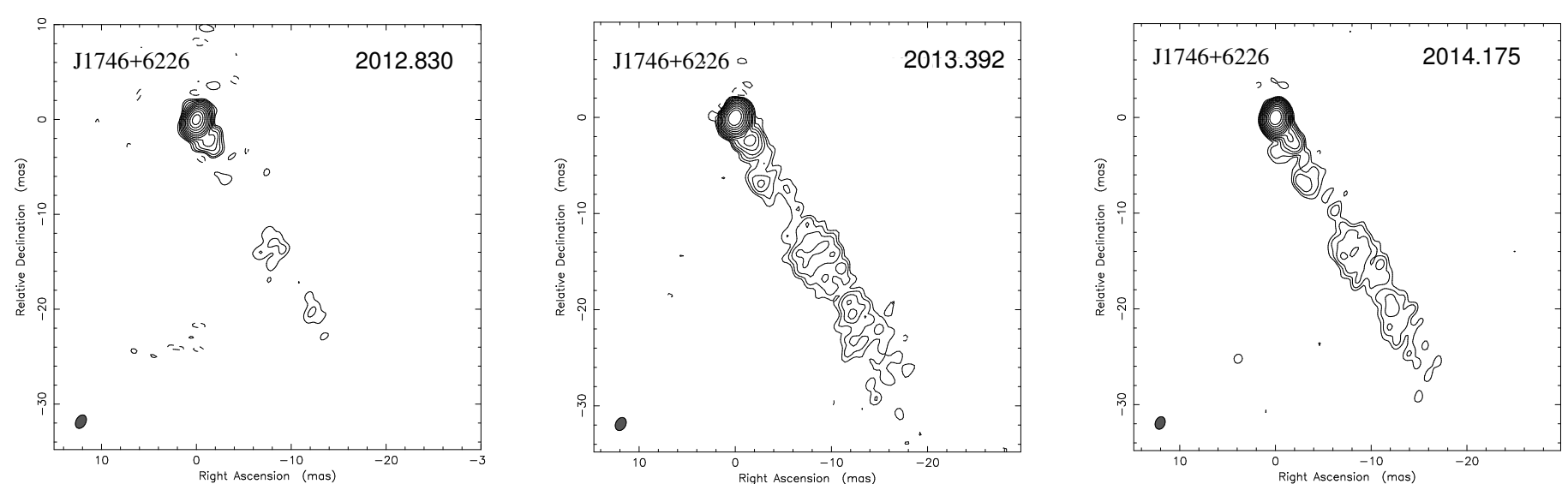

Fig. 1. Maps of J1746+6226 (calibrator of HD 160934) in the three epochs. In each map, the lowest contour levels correspond to 4 times the statistical rms noise $\left(0.5,0.2\right.$, and $\left.0.2 \mathrm{mJy} \mathrm{beam}^{-1}\right)$ with a scale factor between contiguous contours of $\sqrt{3}$. The peak flux densities in the images are $0.32,0.32$, and $0.34 \mathrm{mJy}_{\text {beam }}^{-1}$, respectively. The different morphology in epoch 2012.830 is due to the larger rms.

Table 3. Binary properties of HD 160934.

\begin{tabular}{|c|c|c|c|c|}
\hline $\begin{array}{l}\text { Date } \\
\text { (Filter) }\end{array}$ & Separation & $\begin{array}{l}\mathrm{PA} \\
{\left[{ }^{\circ}\right]}\end{array}$ & Flux ratio & Reference \\
\hline $\begin{array}{c}\text { 30 Jun. } 1998 \\
\text { (F165M) }\end{array}$ & $0.155 \pm 0.001$ & $275.5 \pm 0.2$ & $0.485 \pm 0.006$ & Hormuth et al. (2007) \\
\hline $\begin{array}{l}17 \text { Apr. } 2005 \\
\text { (NIRI-CH4) }\end{array}$ & $0.218 \pm 0.002$ & $268.5 \pm 0.7$ & $0.455 \pm 0.021$ & Lafrenière et al. (2007) \\
\hline $\begin{array}{l}8 \text { Jul. } 2006 \\
\text { (RG830) }\end{array}$ & $0.215 \pm 0.002$ & $270.9 \pm 0.3$ & $0.329 \pm 0.051$ & Hormuth et al. (2007) \\
\hline $\begin{array}{l}19 \text { Nov. } 2015 \\
\left(\text { SDSS } z^{\prime}\right)\end{array}$ & $0.23 \pm 0.01$ & $267 \pm 1$ & $0.5 \pm 0.1$ & This work \\
\hline $\begin{array}{l}19 \text { Nov. } 2015 \\
\left(\text { SDSS } i^{\prime}\right)\end{array}$ & $0.21 \pm 0.03$ & $270 \pm 5$ & $0.6 \pm 0.2$ & This work \\
\hline
\end{tabular}

Notes. The central wavelength of the filters are (in $\mu \mathrm{m}$ ): 1.6511 (F165M), 1.58 (NIRI-CH4), 0.910 (RG830), 0.907 (SDSS $z^{\prime}$ ), and 0.767 (SDSS $i^{\prime}$ ).

Table 4. Compilation of astrometric measurements for the HD 160934 system.

\begin{tabular}{|c|c|c|c|c|}
\hline \multicolumn{5}{|c|}{ Relative positions of HD 160934 A - HD 160934 c } \\
\hline Epoch & Instrument & $\Delta \alpha(\operatorname{mas})$ & $\Delta \delta($ mas $)$ & Reference \\
\hline 1998.496 & HST/NICMOS (IR) & $154.3 \pm 0.9$ & $-14.8 \pm 0.5$ & $(1)$ \\
\hline 2005.296 & Gemini (IR) & $212.9 \pm 2.0$ & $5.6 \pm 2.6$ & (2) \\
\hline 2006.518 & AstraLux (IR) & $214.9 \pm 1.0$ & $-3.4 \pm 1.0$ & (1) \\
\hline 2006.712 & Gemini (IR) & $217.9 \pm 2.0$ & $-4.9 \pm 2.6$ & (2) \\
\hline 2008.477 & Palomar (IR) & $-169.1 \pm 0.3$ & $-9.7 \pm 0.3$ & (3) \\
\hline 2010.318 & Keck (IR) & $64.6 \pm 0.3$ & $-18.9 \pm 0.3$ & (3) \\
\hline 2011.310 & Keck (IR) & $-6.3 \pm 0.3$ & $-18.9 \pm 0.3$ & (3) \\
\hline 2012.830 & EVN (radio) & $-22.3 \pm 0.1$ & $6.2 \pm 0.2$ & (4) \\
\hline 2014.175 & EVN (radio) & $149.3 \pm 0.1$ & $12.9 \pm 0.2$ & (4) \\
\hline 2015.887 & AstraLux (IR) & $225.7 \pm 3.0$ & $11.8 \pm 3.0$ & (4) \\
\hline \multicolumn{5}{|c|}{ Absolute positions of HD $160934(\mathrm{EVN})$} \\
\hline Epoch & Component & $\mathrm{RA}(\mathrm{h} \mathrm{m} \mathrm{s})$ & $\operatorname{Dec}\left({ }^{\circ \prime \prime \prime}\right)$ & \\
\hline \multirow[t]{2}{*}{2012.830} & $\mathrm{~A}$ & $173839.59830 \pm 0.00014$ & $611416.6077 \pm 0.0005$ & (4) \\
\hline & $\mathrm{c}$ & $173839.60138 \pm 0.00014$ & $611416.6015 \pm 0.0005$ & (4) \\
\hline 2013.392 & A & $173839.60667 \pm 0.00016$ & $611416.6865 \pm 0.0007$ & (4) \\
\hline \multirow[t]{2}{*}{2014.175} & A & $173839.61159 \pm 0.00013$ & $611416.6882 \pm 0.0005$ & (4) \\
\hline & $\mathrm{c}$ & $173839.59090 \pm 0.00013$ & $611416.6753 \pm 0.0005$ & (4) \\
\hline
\end{tabular}

Notes. (1) Hormuth et al. (2007); (2) Lafrenière et al. (2007); (3) Evans et al. (2012); (4) this work. The standard deviation of the relative position corresponds to the S/N-based uncertainty of the peaks of brightness of HD $160934 \mathrm{~A}$ and $\mathrm{c}$. The absolute positions were obtained with reference to the IERS coordinate of the external quasar J1746+6226 $\left(\alpha=17^{\mathrm{h}} 46^{\mathrm{m}} 14 \mathrm{~s} 034, \delta=62^{\circ} 26^{\prime} 54^{\prime \prime}\right.$.738). The standard deviation of the absolute position includes, in addition to the uncertainty of the peak of brightness, the contribution of the propagation media and the reference source structure. 
R. Azulay et al.: Young, active radio stars in the AB Doradus moving group
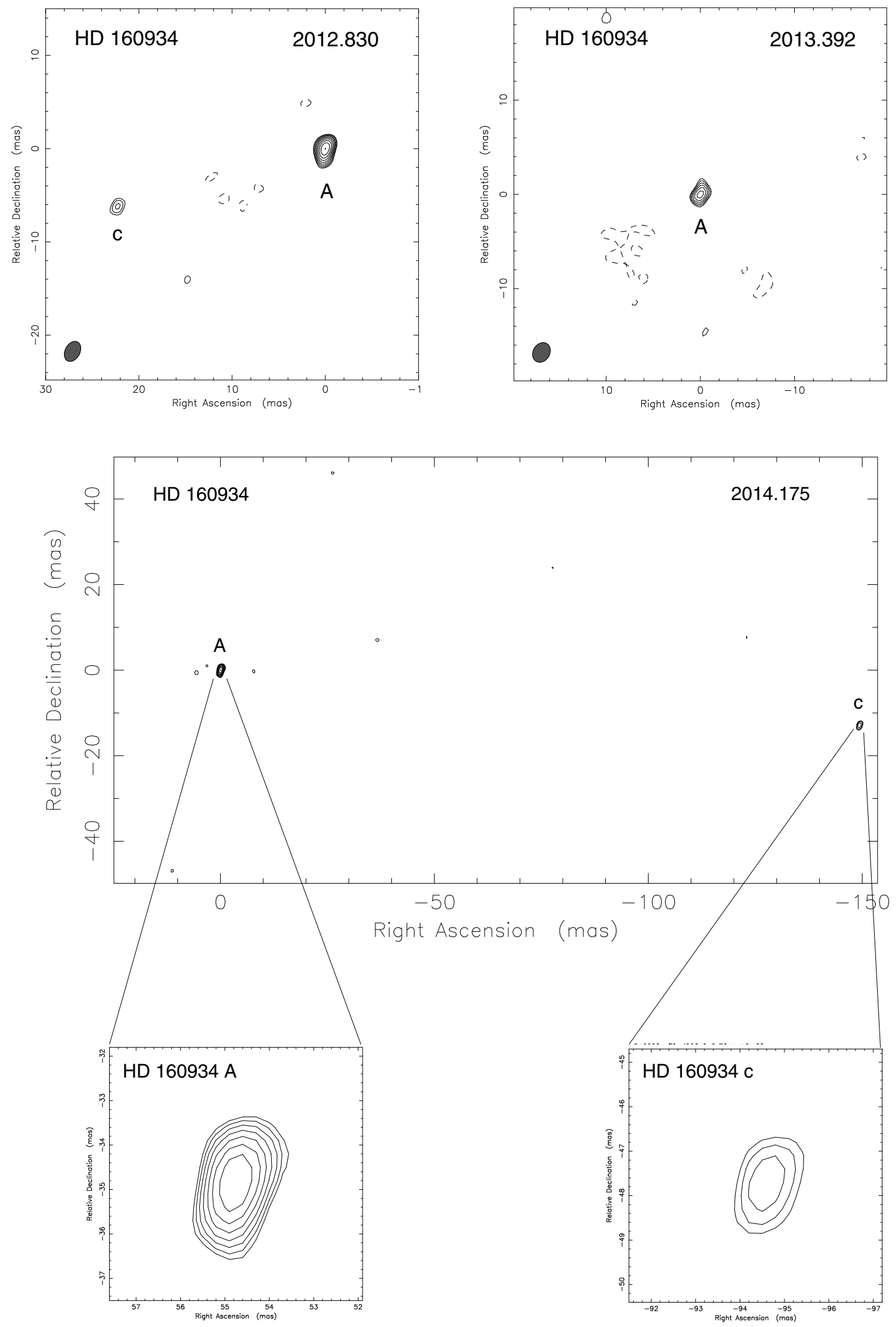

Fig. 2. Clean maps of the binary HD 160934 at the three EVN epochs. The lowest contour levels corresponds to 3 times the statistical rms noise $\left(0.04,0.02\right.$, and $\left.0.04 \mathrm{mJy}_{\text {beam }}{ }^{-1}\right)$ with a scale factor between contiguous contours of $\sqrt{2}$. The peak flux densities in the images are $0.15,0.04$, $0.12 \mathrm{mJy}_{\text {beam }}{ }^{-1}$, respectively. For image parameters see Table 1 . In all the maps we set the origin at the position of the peak of brightness of HD 160934 A. Component $\mathrm{c}$ is not detected at epoch 2013.392. Orbital motion of c around A is evident from epochs 2012.830 and 2014.175. 

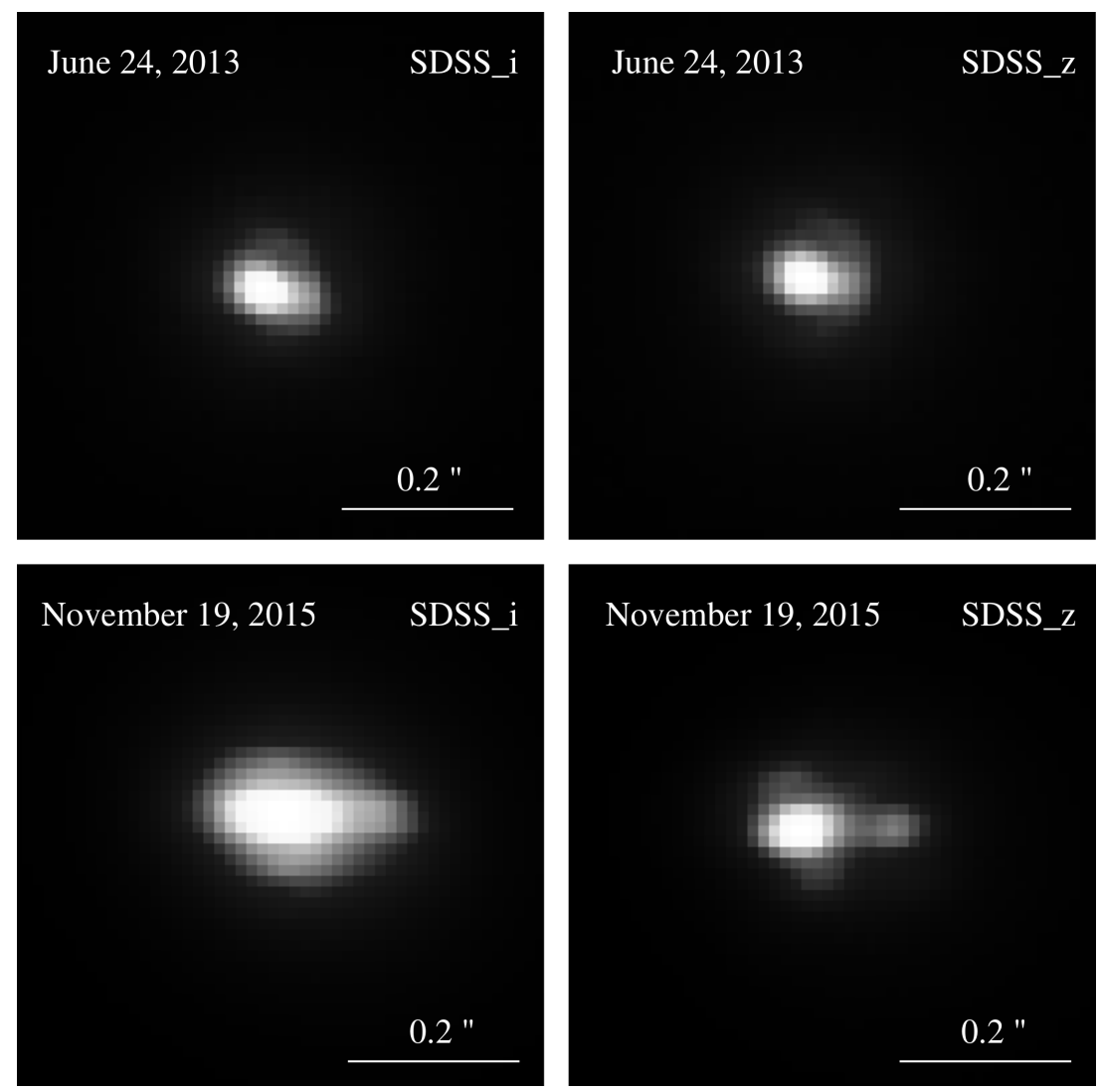

Fig. 3. AstraLux SDSS $i^{\prime}$ and SDSS $z^{\prime}$ images of the binary star HD 160934 in 2013 and 2015; north is up and east is to the left. Both components of the star are not clearly distinguishable in the 2013 images but they are in the 2015 images.

eccentricity $e(0.63)$, three orientation angles $i$ (82:4), $\omega$ (85.9), $\Omega\left(35^{\circ}\right)$, and time of periastron $T_{0}(2002.32)$.

2. We used the values above as (otherwise excellent) a priori to favor the convergence of the L-M algorithm in the combined fit of the absolute (A component) and relative positions $(\mathrm{A}-\mathrm{c})$. In this analysis, the proper motion and parallax of the system were also estimated. The resulting set of astrometric and orbital parameters is shown in Table 5, and meanwhile plots of the relative and absolute orbits are presented in Figs. 4 and 5.

Although there is a third object in this system, HD $160934 \mathrm{~B}$ (located at 8 " separation from A and c; Lowrance et al. 2005), we did not include secular acceleration terms in the fit described above, since this third body does not induce an appreciable acceleration in our three-year time baseline of VLBI monitoring; the estimated period of the corresponding reflex orbital motion is on the order of $10^{3} \mathrm{yr}$ ).

Our fit yielded a new value of the parallax (31.4 \pm 0.5 mas), which is within the uncertainties, but more precise than the previous HIPPARCos estimate (30.2 \pm 2 mas; van Leeuwen 2007). This new parallax allowed us to determine the sum of the masses of both components of HD 160934 using Kepler's third law

$\frac{\left(a_{r e l}^{\prime \prime} / \pi^{\prime \prime}\right)^{3}}{P^{2}}=\left(m_{\mathrm{A}}+m_{\mathrm{c}}\right)_{\odot}$

We obtained a value of $m_{\mathrm{A}}+m_{\mathrm{c}}$ of $1.15 \pm 0.10 M_{\odot}$, which is coincident with previous estimates made by other authors (Evans et al. 2012, and references therein). Similarly, using the semimajor axis of the absolute orbit of component $\mathrm{A}, a_{\mathrm{A}}$, we could estimate the mass of component c, $m_{\mathrm{c}}$, using $m_{\mathrm{c}}^{3} /\left(m_{\mathrm{A}}+m_{\mathrm{c}}\right)^{2}=$ $a_{\mathrm{A}}^{3} / P^{2}$, which yielded a value of $0.45 \pm 0.04 M_{\odot}$. A value of the
Table 5. Estimates of the astrometric and orbital parameters of HD 160934.

\begin{aligned} \hline \hline Parameter & Value \\ \hline$\alpha_{0}(\mathrm{~h} \mathrm{~m} \mathrm{~s}): & 173839.6349 \pm 0.0002 \\ \delta_{0}\left({ }^{\circ}{ }^{\prime \prime}\right): & +611416.0238 \pm 0.0015 \\ \mu_{\alpha}\left(\mathrm{s} \mathrm{yr}^{-1}\right): & -0.0025 \pm 0.0002 \\ \mu_{\delta}\left(\operatorname{arcsec~yr}{ }^{-1}\right): & 0.0469 \pm 0.0002 \\ \pi(\operatorname{arcsec})^{a}: & 0.0314 \pm 0.0005 \\ P(\mathrm{yr}): & 10.26 \pm 0.08 \\ a_{\mathrm{rel}}\left({ }^{\prime \prime}\right): & 0.1554 \pm 0.0008 \\ a_{\mathrm{A}}\left({ }^{\prime \prime}\right): & 0.0603 \pm 0.0014 \\ a_{\mathrm{c}}\left({ }^{\prime \prime}\right): & 0.0952 \pm 0.0014 \\ e: & 0.64 \pm 0.03 \\ i\left(^{\circ}\right): & 82.72 \pm 0.12 \\ \omega_{\mathrm{c}}\left({ }^{\circ}\right)^{b}: & 37.7 \pm 0.5 \\ \Omega\left({ }^{\circ}\right): & 266.74 \pm 0.12 \\ T_{0}: & 2002.4 \pm 0.1 \\ & \\ m_{\mathrm{A}}\left(M_{\odot}\right): & 0.70 \pm 0.07 \\ m_{\mathrm{c}}\left(M_{\odot}\right): & 0.45 \pm 0.04 \\$\hline & \end{aligned}

Notes. The reference epoch is 2000.0. The number of degrees of freedom of the fit is 14; the minimum value found for the reduced $\chi^{2}$ is 1.2 . (a) Our parallax estimate is more accurate than the HIPPARCos value given for $\mathrm{AB}$ Dor A but fully compatible with that within the uncertainty. ${ }^{(b)}$ For the absolute orbit of HD $160934 \mathrm{~A}, \omega_{\mathrm{A}}=\omega_{\mathrm{c}}+\pi$.

$m_{\mathrm{A}}\left(=0.70 \pm 0.07 M_{\odot}\right)$ follows from a simple subtraction of the values above. In principle, the latter value for $m_{\mathrm{A}}$ may seem highly correlated with c; however, a similar, but coarser, estimate 
R. Azulay et al.: Young, active radio stars in the AB Doradus moving group

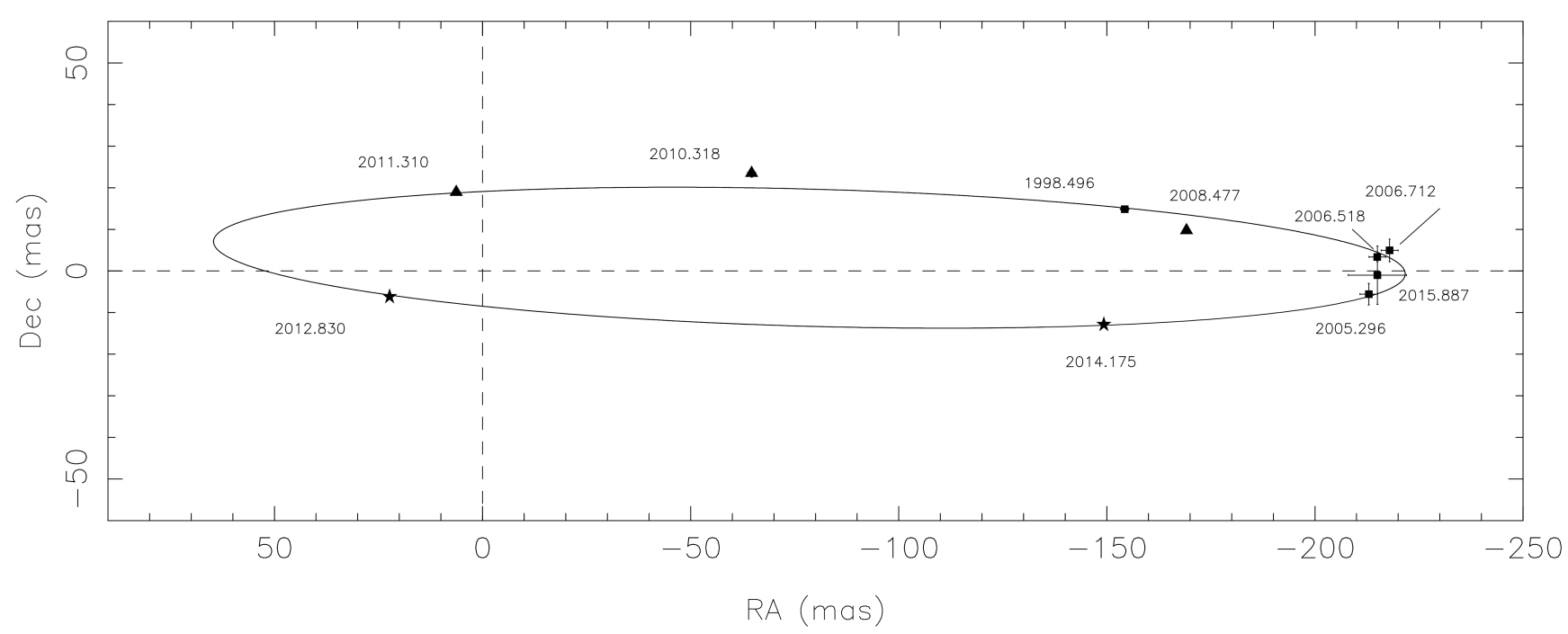

Fig. 4. Relative orbit of the binary star HD 160934 using the orbital elements in Table 5 (with $a_{\text {rel }}$ ). HD $160934 \mathrm{~A}$ is located at the origin of the figure, hence the relative orbit of HD $160934 \mathrm{c}$ is depicted. Each type of symbol corresponds to a different technique to measure the relative position of HD 160934 c, namely, infrared relative astrometry (squares; Hormuth et al. 2007; Lafrenière et al. 2007; this work), masking interferometry (triangles; Evans et al. 2012), and VLBI (star symbol; this work). Error bars are plotted but hardly visible because of the size of the orbit. For clarity, these are shown in Fig. 6.

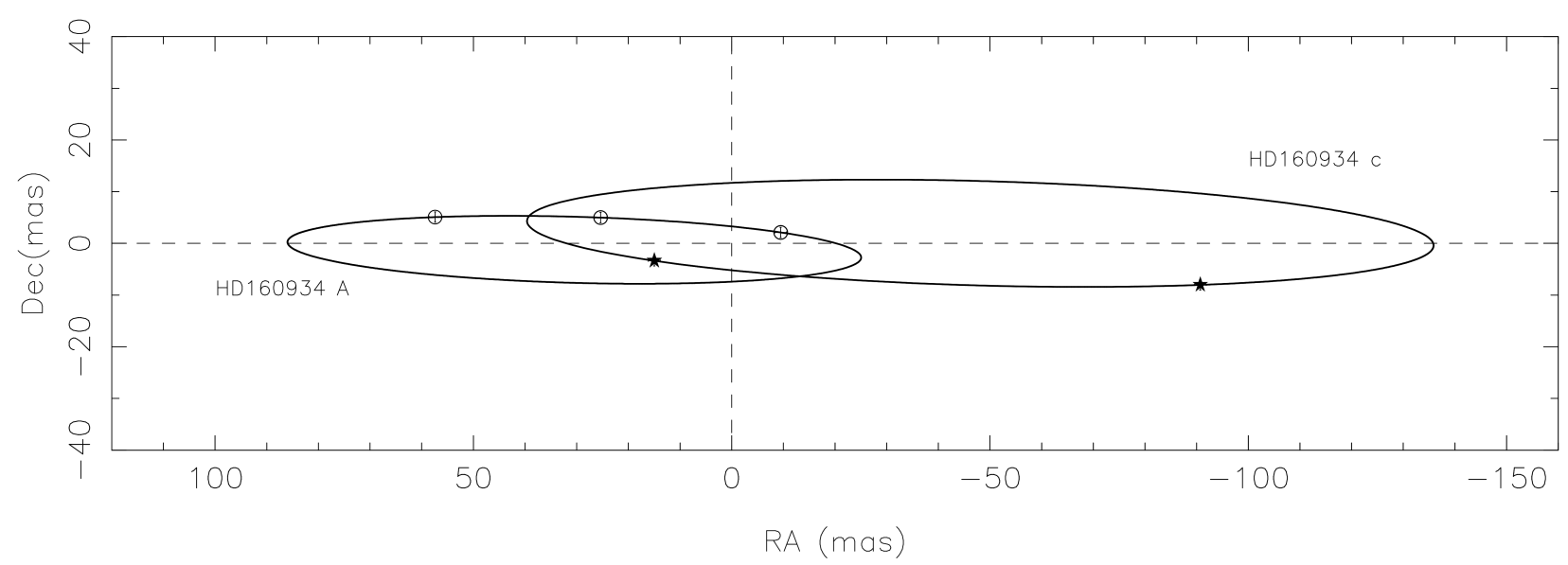

Fig. 5. Absolute orbits of the binary components HD 160934 A and HD $160934 \mathrm{c}$ using the orbital elements in Table 5 (with $a_{\mathrm{A}}$ and $a_{\mathrm{c}}$, respectively). The positions of the component A (circles) and c (star symbols) are indicated. The center of mass of the system is placed at the origin.

of $a_{\mathrm{c}}$ could be obtained by repeating the combined fit using the absolute positions of component c (see Table 4), from which a similar value of $m_{\mathrm{A}}\left(0.70 \pm 0.10 M_{\odot}\right)$ could be calculated. The coincidence of both estimates of $m_{\mathrm{A}}$ indicates the robustness of our mass determinations.

The weighted rms of the postfit residuals (plotted in Fig. 6) is 3.2 mas, meaning that some unmodeled effects are still present in the data. The residuals show no evidence of another companion within the errors. Instead, the possible departure of some of the points from the fitted orbit might indicate instrumental effects that have not been considered. Accordingly, we scaled the statistical errors of the orbital parameters to take this contribution into account (see Table 5).

\subsubsection{Comparison with models}

To proceed with the calibration of PMS models we needed the $K$ magnitude and effective temperature of components A and c. Estimates of the individual $K$ magnitudes can be obtained from the unresolved $J H K 2$ MASS photometry, that is $6.812 \pm 0.020$, combined with the flux ratio between $\mathrm{c}$ and $\mathrm{A}\left(f_{\mathrm{c} / \mathrm{A}}\right)$ at $K$ band; however, although $f_{\mathrm{c} / \mathrm{A}}$ has been measured for different filters (see Table 3 ), the $K$-band magnitude is not available. We estimated $f_{\mathrm{c} / \mathrm{A}}$ at $K$ band via weighted linear fits to the flux ratios measured at other filters given in Table 3 . We obtained a value of $f_{\mathrm{c} / \mathrm{A}}=0.39 \pm 0.11$ as a mean of our fits, and the standard deviation as the spread in the fits considering different subsets of data points, in such a way that our uncertainty conservatively covers the different fitted values of $f_{\mathrm{c} / \mathrm{A}}$. Final values for the absolute $K$ magnitudes were $4.65 \pm 0.15$ and $5.68 \pm 0.15$ for components $\mathrm{A}$ and $\mathrm{c}$, respectively.

Regarding the effective temperatures, we derived a value for component A from its spectral type (K7-K8; McCarthy \& White 2012) using the empirical color-temperature transformation reported by Hartigan et al. (1994). For component c, we proceeded in a similar way by assuming a M2-3 spectral type (Gálvez et al. 2006). The final values for the effective temperatures were $3960 \pm 60 \mathrm{~K}$ and $3450 \pm 90 \mathrm{~K}$ for components $\mathrm{A}$ and $\mathrm{c}$, respectively. The standard deviation of these temperatures were estimated to cover several uncertainties that could affect our approach. Recent studies have shown that the spectral types of young stars determined from NIR observations disagree with 

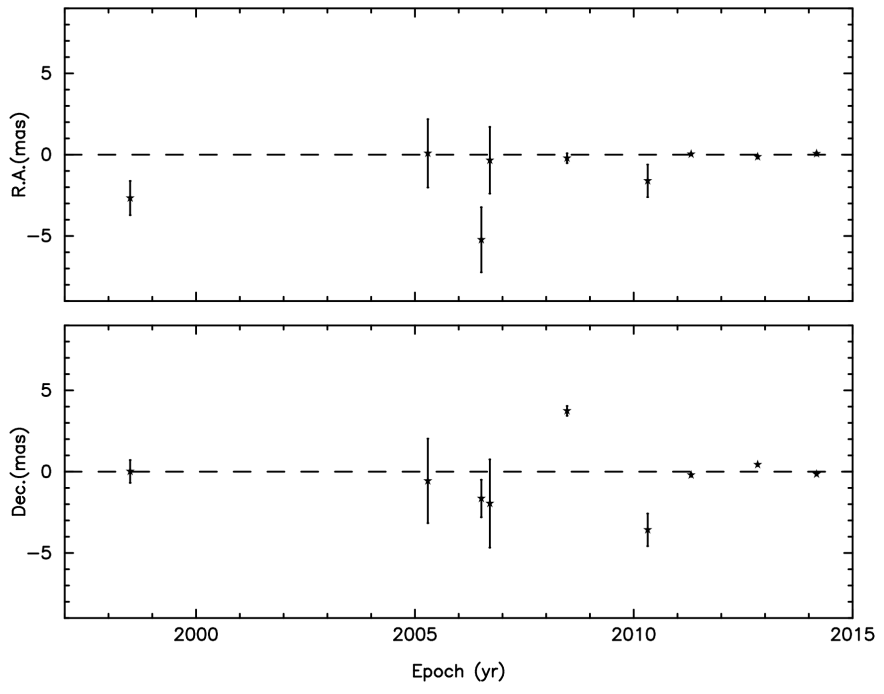

Fig. 6. Postfit residuals in right ascension (upper panel) and declination (lower panel). The weighted rms of the plotted residuals is 3.2 mas.

those from the optical by up to three subtypes (Kastner et al. 2015; Pecaut et al. 2016), which would strongly affect the procedure to estimate the effective temperature. Since the spectral types of HD 160934 A and c are based on IR observations, we should not expect such large errors. Actually, the spectral type determination used for the components of HD 160934 (see above) are 1 subtype uncertain, which has been taken into account to estimate the standard deviation of the effective temperatures. Considering that these standard deviations have been further enlarged to cope with the different, more recent, colortemperature relations (Pecaut \& Mamajek 2013; Luhman et al. 2003; Herczeg \& Hillenbrand 2014) this wavelength-dependent spectral type effect should be reasonably covered.

In order to calibrate the stellar evolution models for PMS stars, we considered isochrones and isomasses corresponding to the models of Baraffe et al. (1998; BCAH98), Siess et al. (2000; S00), Tognelli et al. (2011, 2012; TDP12), Bressan et al. (2012; Padova), Baraffe et al. (2015; BHAC15), and Choi et al. (2016; MIST). We adopted a metallicity value of $[\mathrm{Fe} / \mathrm{H}]=0.0$ (representative of the AB Dor metallicity; Barenfeld et al. 2013) and a solar calibrated mixing length parameter $\alpha$. The different models can be shown in Fig. 7. HD 160934 A and HD $160934 \mathrm{c}$ are placed in the H-R diagrams in Fig. 7 using the values of both the magnitudes and temperatures explained above.

The theoretical masses predicted by the models agree with our dynamical estimates just at the extreme of their uncertainties. All sets of tracks predict masses for component A $\sim 10 \%$ lower than our dynamical values, while predictions for the component c vary according to the model: BCAH98, BHAC15, and MIST predict masses that are $\sim 30 \%$ lower, S00 and TDP12 predict masses that are $\sim 40 \%$ lower, and Padova predict masses that are $\sim 10 \%$ lower. These results are consistent with previously published works, which conclude that PMS stellar evolution models for low-mass stars underestimate the dynamical values between 10-30\% (Hillenbrand \& White 2004; Mathieu et al. 2007). According to the values above, predictions are better for component $\mathrm{A}$ than for component $\mathrm{c}$, that is, the larger the dynamical mass, the smaller the difference between the theoretical and dynamical estimates.

In terms of age, the S00 and TDP12 models suggest that both stars are younger than $40 \mathrm{Myr}$, meanwhile BCAH98, Padova, BAHC15, and MIST favor slightly older ages but younger than 60 Myr. The age of the HD 160934 system predicted by the models in Fig. 7 is between $15 \mathrm{Myr}$ and $60 \mathrm{Myr}$, near the young estimates of the age of the AB Dor-MG. Age estimates of the AB Dor-MG range from 50-120 Myr (Malo et al. 2013), $70 \mathrm{Myr}$ (Zuckerman et al. 2011), 70-120 Myr (Gagné et al. 2014), 100-150 Myr (Elliott et al. 2016), and 130-200 Myr (Bell et al. 2015). It should be mentioned that the possibility that HD 160934 does not belong to the AB Dor-MG cannot be ruled out. According to the BANYAN II membership probability tool (Gagné et al. 2014), the kinematics of this object favor HD 160934 to be a young field dwarf with a 95\% probability. Were this the case for HD 160934, the conclusions about the age of the AB Dor-MG based on the age range derived from Fig. 7 would have a limited validity. However, in contrast with the BANYAN prediction, a very recent publication (Elliott et al. 2016) still confirms HD 160934 as an AB Dor-MG member.

\subsubsection{Magnetic field effects}

The discrepancy between the dynamical and the inferred mass for the components of HD 160934 seems to show the lack of additional input physics in the models. Indeed, the models appear to be too hot for the data at a given mass. To this regard, several hypothesis exist that would allow the models to be cooler; in the case of HD 160934, and given the existence of compact radio emission on both objects, which is frequently associated with intense magnetic activity (i.e., Güdel et al. 1995), we limited our analysis to study the impact of a magnetic field in the stellar models.

The presence of a magnetic field inside the star mainly acts to reduce the surface convection efficiency in stellar models, as shown in Feiden et al. (2012, 2013, 2014, 2016), thus reducing the stellar effective temperature, and increasing the star's radius. Moreover, an external magnetic field might also result in surface spots that, blocking the flux at the stellar surface, tend to increase the stellar radius (Somers \& Pinsonneault 2015). Thus, we briefly analyze these two aspects in turn.

Regarding the internal magnetic field and following Feiden et al. (2013), its main effect on the convective heat transport can be partially simulated by reducing the efficiency of superadiabatic convection, which is effectively carried out using a value of the mixing-length parameter $\alpha$ that is much lower than the solar calibrated value. Consequently, we computed new TDP12 models using a value of $\alpha=0.6$, in contrast with the solar calibrated $\alpha=1.74$ value used in the models of Fig. 7. This particular value of $\alpha$ has been adopted by Feiden et al. (2013) to simulate the reduced convection efficiency due to a magnetic field in a non-magnetic stellar model; also such a low $\alpha$ value is compatible with those used by Chabrier et al. (2007) to reproduce the radius of low-mass eclipsing binaries stars. The results are shown in Fig. 8 (left), where we see an evident "cooling" of the magnetic $(\alpha=0.6)$ isomasses of both components $\mathrm{A}$ and c with respect to those corresponding to the standard models ( $\alpha=1.74$; see Fig. 7), which in turn produces a better agreement with the measurements. Interestingly, the effect of the internal magnetic field leads to older ages for both A (>50 Myr) and c (>30 Myr) components.

Concerning the effect of surface spot coverage on the models, we computed additional TDP12 evolutionary models, in which we implemented this effect following the formalism described in Somers \& Pinsonneault (2015). We adopted two different values of the effective spot coverage $\beta$ of 0 (standard models without spots; Fig. 7) and 0.3, which is the same value used by Somers \& Pinsonneault (2015). The comparison can be 
R. Azulay et al.: Young, active radio stars in the AB Doradus moving group
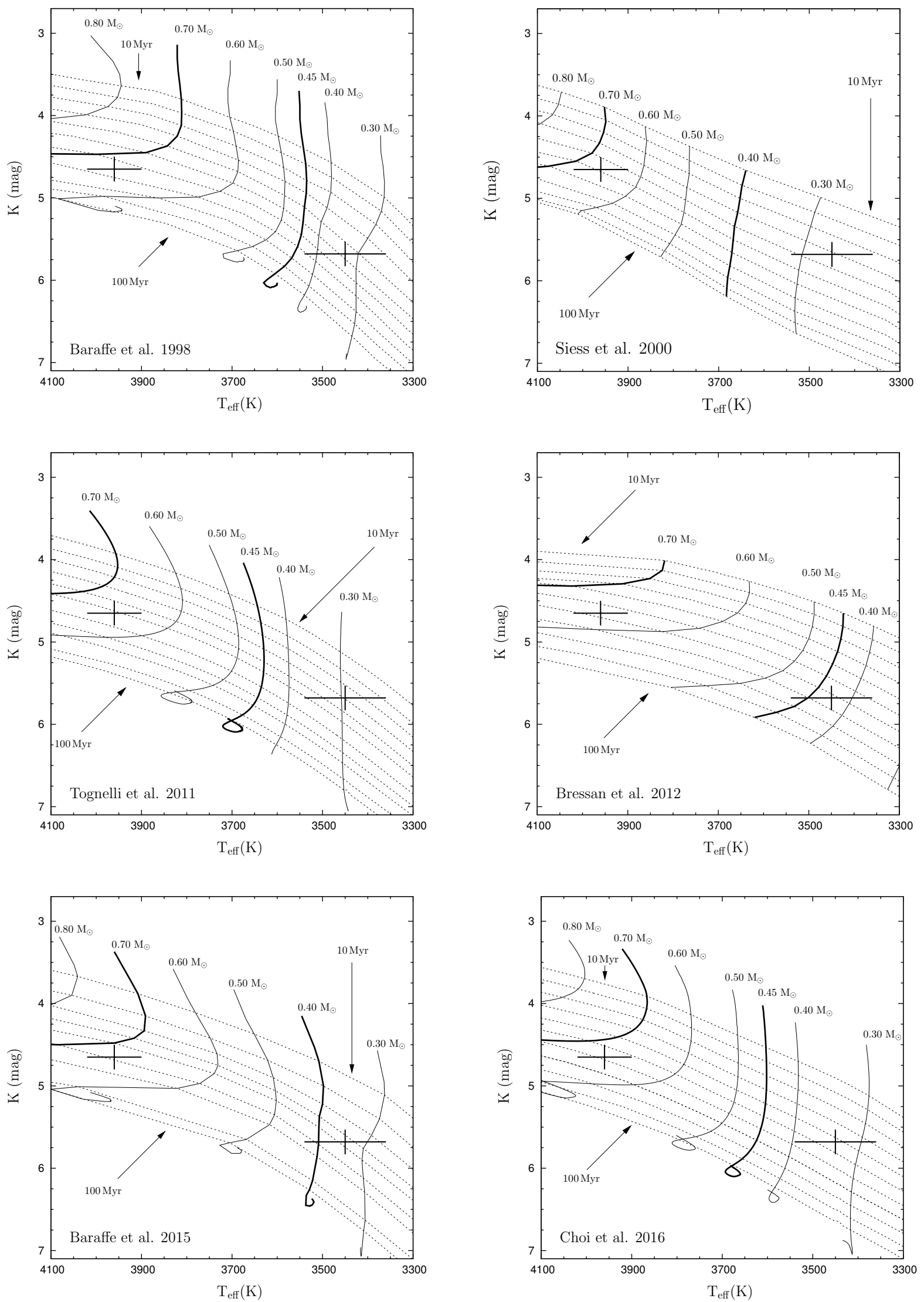

Fig. 7. Comparison of HD 160934 components with some PMS theoretical models (Baraffe et al. 1998, top left; Siess et al. 2000, top right; Tognelli et al. 2011, middle left; and Bressan et al. 2012, middle right; Baraffe et al. 2015, bottom left; and Choi et al. 2016, bottom right). For each model, isomasses (solid lines) and isochrones (dashed lines) are plotted. We highlight the nearest tracks available corresponding to our dynamical mass values. The theoretical masses are consistent with our dynamical estimates just at the extreme of their uncertainties. 

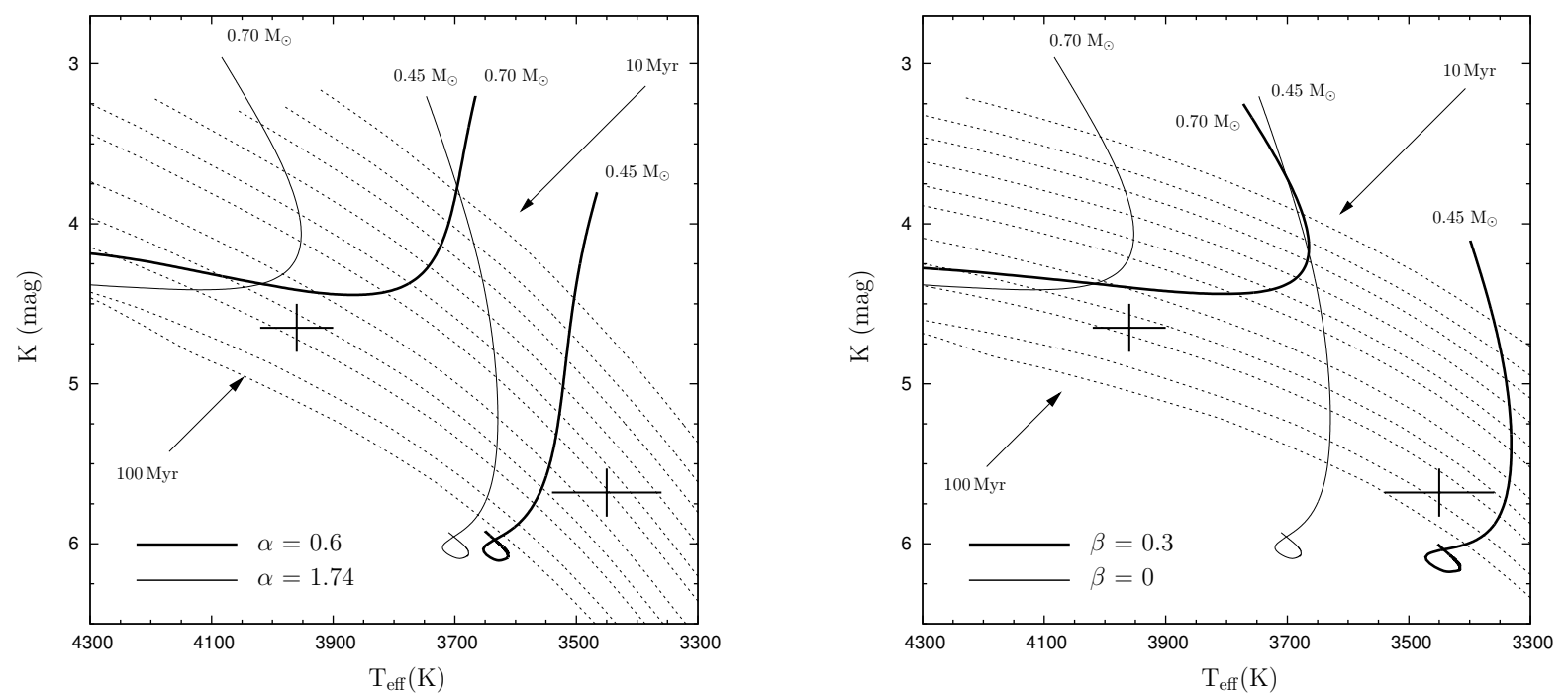

Fig. 8. TPD12 models recalculated to include the effects of the stellar magnetic field. Left: effect of an internal magnetic field, simulated by using a value of $\alpha=0.6$. The isomasses of the solar value $\alpha=1.74$ are also plotted for comparison. Right: effect of stellar spots, computed for an effective spot coverage of $\beta=0.3$ (Somers \& Pinsonneault 2015). The isomasses for $\beta=0$ (standard models with no spots) are also plotted for comparison. In both cases, the isochrones (dashed lines) of the altered models are also shown and are to be compared with the isochrones of the TPD12 models in Fig. 7. See text.

seen in Fig. 8 (right), where we see similar effects to those shown for the internal magnetic field: cooling of the isomasses, leading to a reduction of the discrepancy with the measurements, and older ages ( $>50 \mathrm{Myr}$ ) for both components.

There are other possible scenarios that can modify the position of a pre-MS star in the HR diagram, such as the presence of protostellar accretion, but its treatment is beyond the scope of this paper; in such a scenario, it is difficult to define a standard set of accretion models, as the outputs of the models are severely affected by the parameters that govern the accretion phase (e.g., mass accretion rate, initial seed mass, or thermal energy carried inside the star by the accreted matter; Baraffe et al. 2012; Tognelli et al. 2015).

\subsection{EKDraconis}

EK Dra (=HD 129333) is an active, G1.5 V star with a rapid rotation (2.6 days; Järvinen et al. 2005). The binarity of this object (whose components are EK Dra A/B, separated by $0.74^{\prime \prime}$ ) was discovered for the first time through radial velocity variations by Duquennoy \& Mayor (1991). Metchev \& Hillenbrand (2004) confirmed the existence of these components from IR imaging. Several radial velocity studies of this star have been carried out (Duquennoy \& Mayor 1991; Dorren \& Guinan 1994; Montes et al. 2001; König et al. 2005). In particular, Köning et al. (2005) combined these radial velocity data with their data of speckle interferometry to derive masses of $0.9 \pm 0.1 M_{\odot}$ and $0.5 \pm 0.1 M_{\odot}$, for the primary and secondary, respectively, a period of $45 \pm 5 \mathrm{yr}$, and a semimajor axis of $14.0 \pm 0.5 \mathrm{AU}$.

The VLA image (Fig. 10) shows EK Dra as an unresolved radio emitter with an integrated flux of $0.21 \mathrm{mJy}$ (the radio emission of EK Dra A at radio wavelengths is known and reported in Güdel et al. 1995). Because of the small separation of both components of the binary at the epoch of observation, the components $\mathrm{A}$ and $\mathrm{B}$ appear to be blended on the map. Besides this, we could only detect the component A in the first of our three VLBI epochs of observations (2012.827). The image yields a flux density of $0.06 \mathrm{mJy}$, with an upper bound to the radio emission of the component B of $0.02 \mathrm{mJy}$ (Fig. 11). The upper bounds to the radio emissions of the star in the second and third epochs are 0.01 and $0.02 \mathrm{mJy}$, respectively. The non-detection of EK Dra in the last two VLBI epochs can be a consequence of the variable behavior of the radio emission. In the AstraLux images (Fig. 12), meanwhile, we could detect both components of the star.

We used our AstraLux relative position of EK Dra to revisit the orbital motion between components $\mathrm{A}$ and $\mathrm{B}$. This position is shown in Table 6 along with already published relative positions of EK Dra A/B, which mostly results from speckle interferometry observations (König et al. 2005). We performed a weighted least-squares analysis similar to that presented for HD 160934, simplified in this case to deal with only relative positions. Table 7 shows the resulting orbital elements. The estimate of the combined mass of the system, using the HIPPARCOS distance $33.94 \pm 0.72 \mathrm{pc}$, is $m_{\mathrm{A}}+m_{\mathrm{B}}=1.38 \pm 0.08 M_{\odot}$. Plots of the relative orbit can be seen in Figs. 13 and 14. Both the orbital elements and mass estimates coincide with those reported by König et al. (2005) within uncertainties. Although our new position extends twofold the time baseline of the orbital monitoring, the motion of B around the main star is very slow, indicating that the components are near the apoastron.

\subsection{PWAndromedae}

PW And (=HD 1405) is a chomospherically very active star with a spectral type $\mathrm{K} 2 \mathrm{~V}$, which displays a fast rotation (1.75 days) (Montes et al. 2001). Strassmeier et al. (1988) included this object as a possible binary, however, radial velocity studies (Griffin 1992; López-Santiago et al. 2003) discard the presence of a close, interacting companion, showing that the chomospheric activity is due to PW And itself. Moreover, Evans et al. (2012) explored the inner region of the star with speckle interferometry excluding the presence of companions at separations larger than 20 mas.

Regarding the radio observations, a VLA image (Fig. 15) reveals a flux density of $0.34 \mathrm{mJy}$. Our EVN image (Fig. 17), meanwhile, shows an unresolved source that should correspond 
R. Azulay et al.: Young, active radio stars in the AB Doradus moving group
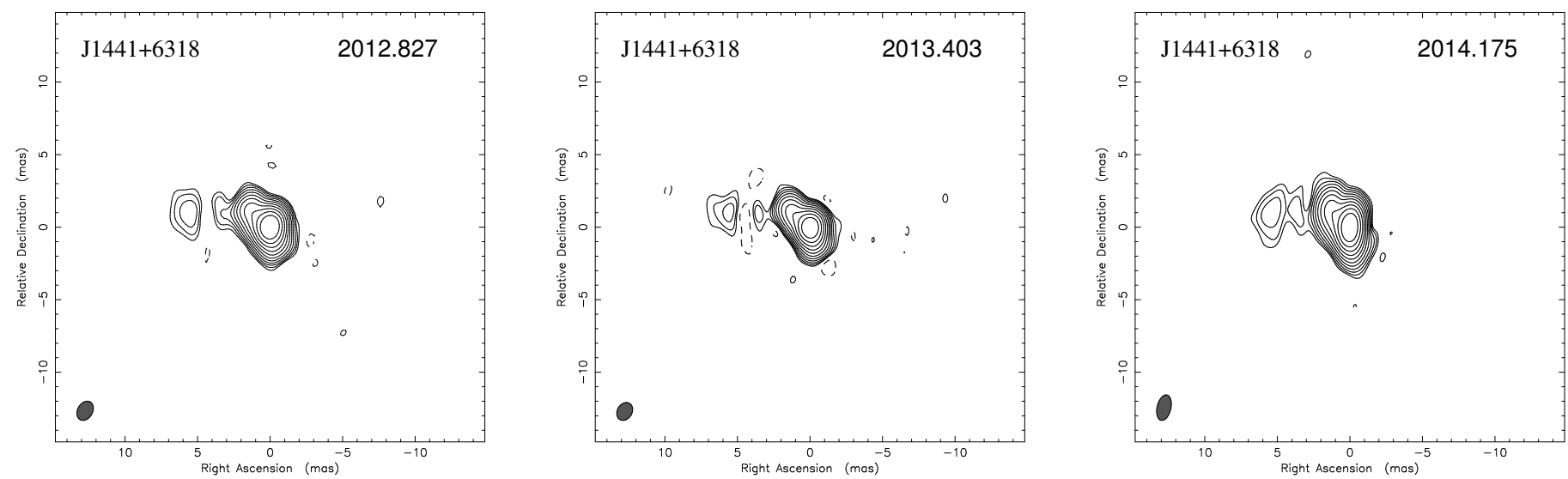

Fig. 9. EVN $5 \mathrm{GHz}$ images of J1441+6318 (calibrator of EK Dra) taken on 2012.827, 2013.403, and 2014.175, respectively. In each map, the lowest contour level corresponds to 5 times the statistical rms noise $\left(0.3,0.4\right.$, and $\left.0.4 \mathrm{mJy} \mathrm{beam}^{-1}\right)$ with a scale factor between contiguous contours of $\sqrt{3}$. The peak flux densities in the images are $0.17,0.17$, and $0.19 \mathrm{mJy}^{-1}$ beam ${ }^{-1}$, respectively.

Table 6. Compilation of astrometric measurements for the EK Dra system.

\begin{tabular}{ccrrr}
\hline \hline \multicolumn{5}{c}{ Relative positions of EK Dra B - EK Dra A } \\
Epoch & Instrument & $\Delta \alpha($ mas $)$ & $\Delta \delta($ mas $)$ & Reference \\
\hline 1991.2135 & 1D & $+0.030 \pm 0.020$ & $-0.280 \pm 0.015$ & $(1)$ \\
1992.1232 & 1D & $+0.045 \pm 0.022$ & $-0.310 \pm 0.015$ & $(1)$ \\
1993.7611 & MAGIC & $+0.050 \pm 0.010$ & $-0.453 \pm 0.015$ & $(1)$ \\
1994.0712 & MAGIC & $+0.051 \pm 0.004$ & $-0.483 \pm 0.010$ & $(1)$ \\
1994.9501 & MAGIC & $+0.040 \pm 0.004$ & $-0.499 \pm 0.005$ & $(1)$ \\
1997.8926 & MAGIC & $+0.059 \pm 0.008$ & $-0.644 \pm 0.012$ & $(1)$ \\
2001.1123 & OMEGA Cass & $+0.085 \pm 0.007$ & $-0.674 \pm 0.005$ & $(1)$ \\
2001.8406 & OMEGA Cass & $+0.074 \pm 0.013$ & $-0.705 \pm 0.012$ & $(1)$ \\
2002.8049 & OMEGA Cass & $+0.102 \pm 0.012$ & $-0.718 \pm 0.009$ & $(1)$ \\
2013.4820 & AstraLux & $+0.081 \pm 0.008$ & $-0.773 \pm 0.008$ & $(2)$ \\
\hline
\end{tabular}

Notes. (1) König et al. (2005) using the $3.5 \mathrm{~m}$ telescope on Calar Alto; (2) this work.

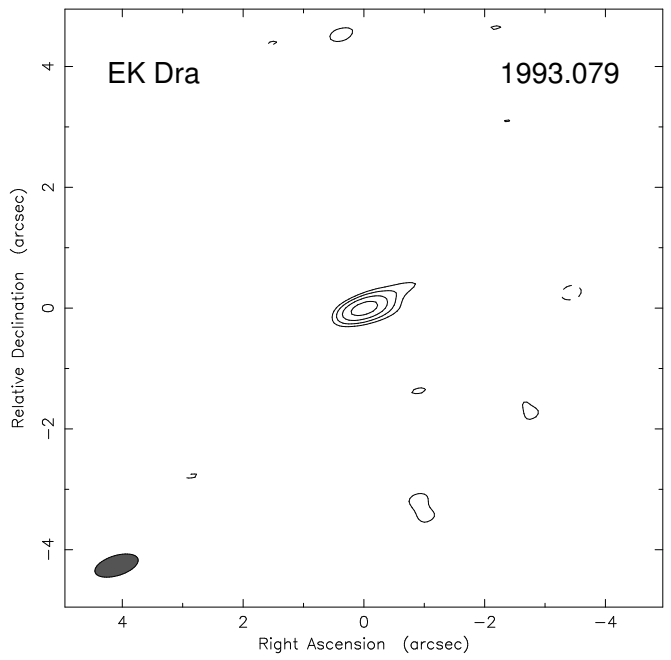

Fig. 10. VLA $8.4 \mathrm{GHz}$ image of EK Dra taken on 1993.079. The lowest contour level corresponds to twice the statistical rms noise $\left(0.02 \mathrm{mJy}\right.$ beam $\left.^{-1}\right)$ with a scale factor between contiguous contours of $\sqrt{2}$. The peak flux density in the image is $0.18 \mathrm{mJy} / \mathrm{beam}$. The restoring beam (shown in the bottom-left corner) is an elliptical Gaussian of $0.74 \times 0.33 \operatorname{arcsec}(\mathrm{PA}-72: 8)$.

to PW And with a flux density of $0.17 \mathrm{mJy}$. Since our EVN observation does not show any companion to PW And, we can

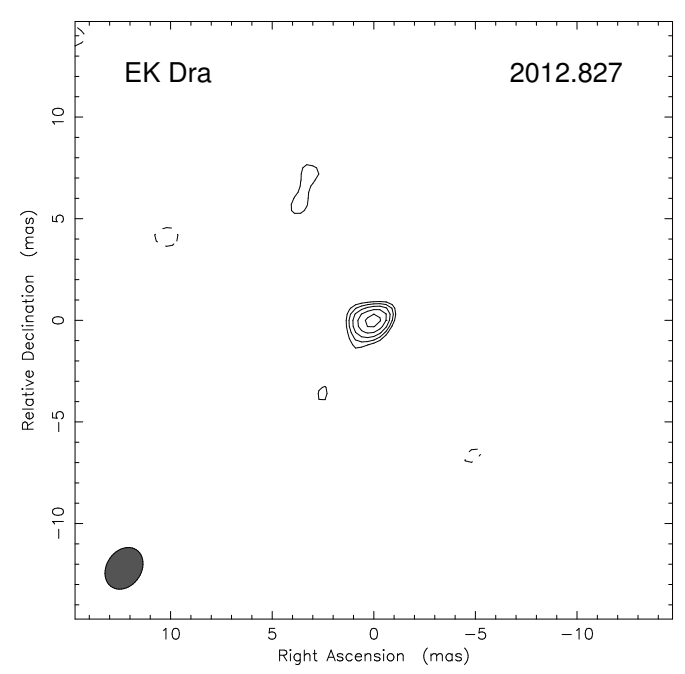

Fig. 11. EVN $5 \mathrm{GHz}$ image of EK Dra taken on 2012.827. The lowest contour level corresponds to 3 times the statistical rms noise $\left(0.02 \mathrm{mJy}\right.$ beam $\left.^{-1}\right)$ with a scale factor between contiguous contours of $\sqrt{2}$. The peak flux density in the image is $0.06 \mathrm{mJy}_{\text {beam }}^{-1}$. The restoring beam (shown in the bottom-left corner) is an elliptical Gaussian of $2.19 \times 1.71$ mas $(\mathrm{PA}-34.1)$.

extend the absence of companions down to the resolution of our array, $\sim 5$ mas, at a flux density limit level of $0.01 \mathrm{mJy}$. Given 

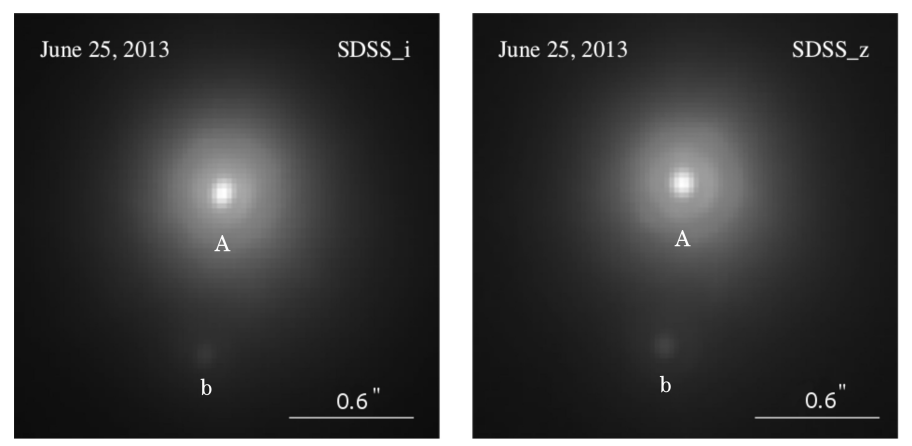

Fig. 12. AstraLux SDSS_i and SDSS_z images of the binary star EK Dra in 2013; north is up and east is to the left. Both components of the star, component A (north) and B (south), are clearly detectable.

Table 7. Estimates of the orbital parameters of EK Dra.

\begin{aligned} & \hline \hline Parameter Value \\ & \hline$P(\mathrm{yr}): 47.9 \pm 0.9 \\ & a_{\text {rel }}\left({ }^{\prime \prime}\right): 0.434 \pm 0.003 \\ & e: 0.812 \pm 0.009 \\ & i\left(^{\circ}\right): 89 \pm 1 \\ & \omega\left(^{\circ}\right): 180 \pm 1 \\ & \Omega\left(^{\circ}\right):-186 \pm 1 \\ & T_{0}: 1986.2 \pm 0.1 \\ &$\hline\end{aligned}

the apparent single character of the star, we did not propose further EVN observations of this source in the frame of this work (dedicated to monitor binary/multiple systems). Still, once confirmed the presence of compact emission, the determination of a precise, VLBI-based parallax value, which supersedes that of HIPPARCOS, might be certainly of interest.

\subsection{LO Pegasus}

LO Peg $(=\mathrm{BD}+224402)$ is a young, active star with spectral type in the range K3V-K8V (Zuckerman \& Song 2004; Pandey et al. 2005) and a rotation period of 0.42 days (Barnes et al. 2005). The first study of LO Peg was carried out by Jeffries et al. (1994), who concluded that there was no circumstellar matter around the star. Since then, Doppler images and studies of radial velocity have been carried out (Barnes et al. 2005; Piluso et al. 2008) and all of these studies consider LO Peg as a single star.

We can confirm the radio emission of this source with a VLA image (Fig. 18) that reveals a flux density of $0.45 \mathrm{mJy}$. In the VLBI image, nevertheless, the star could not be detected. The upper bound to radio emission of LO Peg in this observation is $0.08 \mathrm{mJy} /$ beam. This is because the non-detection could reflect the high variability of these active stars.

\section{Discussion and conclusions}

The main properties of the AB Dor-MG stars studied in this paper are shown in Table 8, including our calculated values of the absolute radio luminosity (obtained from the VLA flux and the HIPPARCos distance) and the brightness temperature (obtained from the VLBI angular size when the source is detected). While $\mathrm{AB}$ Dor $\mathrm{Ba} / \mathrm{Bb}$ and $\mathrm{HD} 160934 \mathrm{~A} / \mathrm{c}$ resulted to be intense radio emitters that were detected at all observing epochs (and so it is PW And, also detected in our unique VLBI epoch of observation), we found that EK Dra (in 2 out 3 epochs) and LO Peg

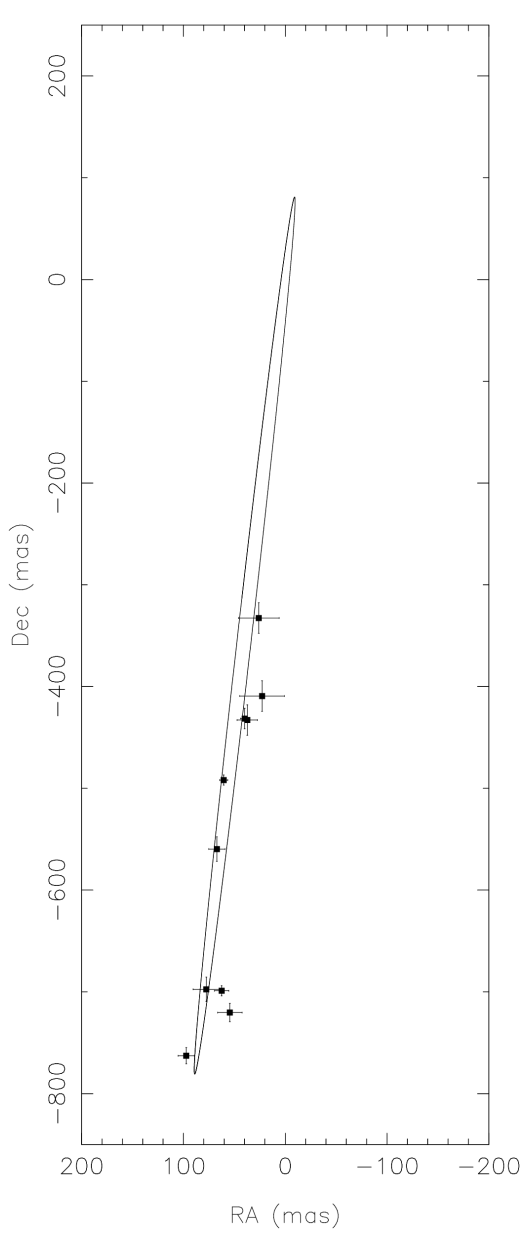

Fig. 13. Relative orbit for the binary EK Dra using the orbital elements in Table 7. EK Dra A component is located at the origin.
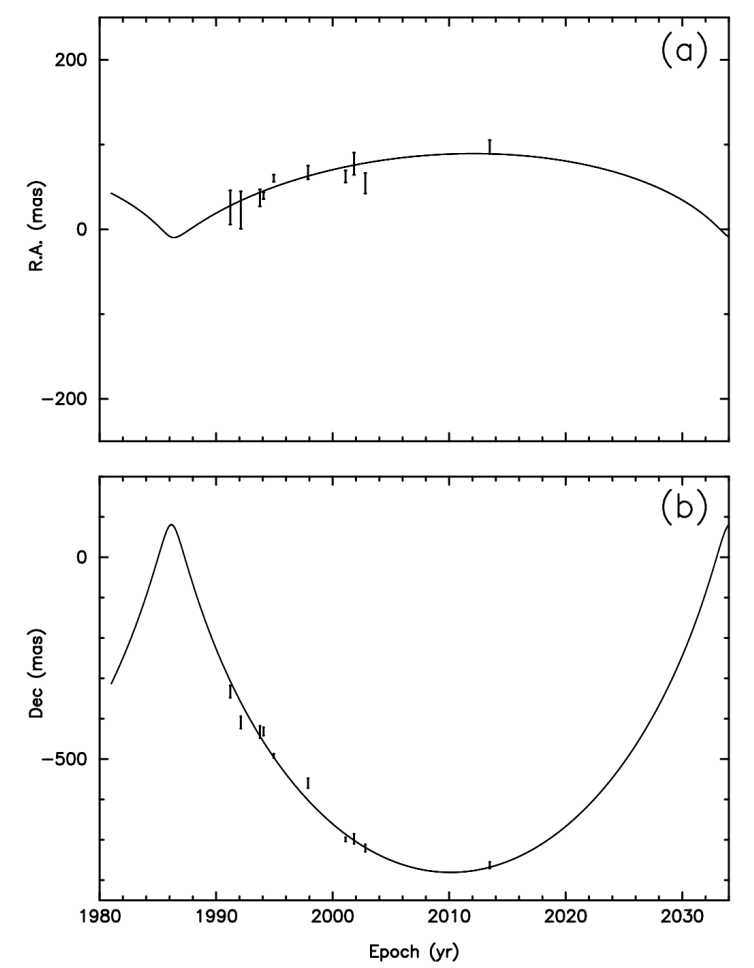

Fig. 14. Orbital motion of the binary EK Dra in right ascension a) and declination b). The line corresponds to the least-squares fitted values of Table 7. 
Table 8. Radio stars from the AB Doradus moving group.

\begin{tabular}{lcccccc}
\hline \hline Name & $\begin{array}{c}T_{\mathrm{b}} \\
\left(\times 10^{6} \mathrm{~K}\right)\end{array}$ & $\begin{array}{c}v \sin i \\
\left(\mathrm{~km} \mathrm{~s}^{-1}\right)\end{array}$ & $\begin{array}{c}P_{\text {rot }} \\
(\mathrm{d})\end{array}$ & $\begin{array}{c}\log L_{\mathrm{X}} \\
(\mathrm{erg} / \mathrm{s})\end{array}$ & $\begin{array}{c}\log L_{\mathrm{R}} \\
(\mathrm{erg} / \mathrm{s} / \mathrm{Hz})\end{array}$ & References \\
\hline PW And & 0.81 & 24 & 1.75 & 30.5 & 14.5 & $1,12,13,14$ \\
AB Dor Ba/Bb & $2.25 / 2.60$ & 9 & 0.33 & - & 14.6 & $2,3,4,5$ \\
EK Dra A/B & $0.73 /-$ & 16.5 & 2.78 & 29.92 & 14.6 & $1,6,7,14$ \\
HD 160934 A/c & $0.72 / 1.4$ & 17 & 1.8 & 29.39 & 14.4 & $2,8,9,10,14$ \\
LO Peg & - & 60 & 0.42 & 30.2 & 14.7 & $11,12,13,14$ \\
\hline
\end{tabular}

References. (1) Montes el al. (2001); (2) Zuckerman \& Song (2004); (3) Lim (1993); (4) Janson et al. (2007); (5) Azulay et al. (2015); (6) Güdel et al. (1995); (7) König et al. (2005); (8) Hormuth et al. (2007); (9) Evans et al. (2012); (10) Azulay et al. (2014); (11) Jeffries et al. (1994); (12) Wichmann et al. (2003); (13) VLA data archive; (14) This work.

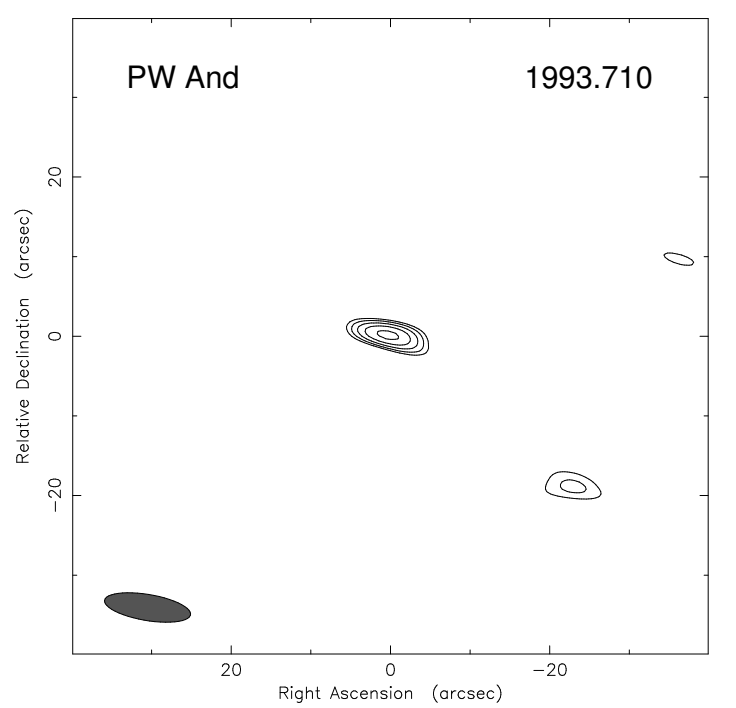

Fig. 15. VLA $8.4 \mathrm{GHz}$ image of PW And taken on 1993.710. The lowest contour level corresponds to 3 times the statistical rms noise $\left(0.02 \mathrm{mJy}\right.$ beam $\left.^{-1}\right)$ with a scale factor between contiguous contours of $\sqrt{2}$. The peak flux density in the image is $0.29 \mathrm{mJy} / \mathrm{beam}$. The restoring beam (shown in the bottom-left corner of the map) is an elliptical Gaussian of $10.90 \times 3.28 \operatorname{arcsec}\left(\mathrm{PA} 80^{\circ} .5\right)$.

did not display detectable levels of radio emission. These nondetections might just reflect the variability of the radio emission, since, as seen in Table 8, neither the distance, rotation period, nor X-ray luminosity are significantly different in these two stars with respect to the other radio emitter systems. Therefore, further monitoring of these non-detected stars would not be as efficient in terms of kinematical studies as in the cases presented for AB Dor Ba/Bb (Azulay et al. 2015) and HD 160934 (this paper). The same conclusion applies to PW And, given its apparent non-binarity (even taking into account a clear VLBI detection).

On the other hand, from the brightness temperatures shown in Table 8, we can conclude that the radio emission has a nonthermal origin. This fact, along with the rapid rotation values and saturated levels of X-ray luminosity, $L_{\mathrm{X}}$, also listed in the table, favors the existence of an intense magnetic activity of the stellar corona that is responsible in terms of the presence of this radio emission. Therefore, the radio emission is apparently generated by gyrosynchrotron emission from non-thermal accelerated electrons (Lim et al. 1994; Güdel et al. 1995).

In this paper we have shown the results of a VLBI program dedicated to monitoring the absolute reflex motion of HD 160934, a member of the AB Dor-MG. The unexpected

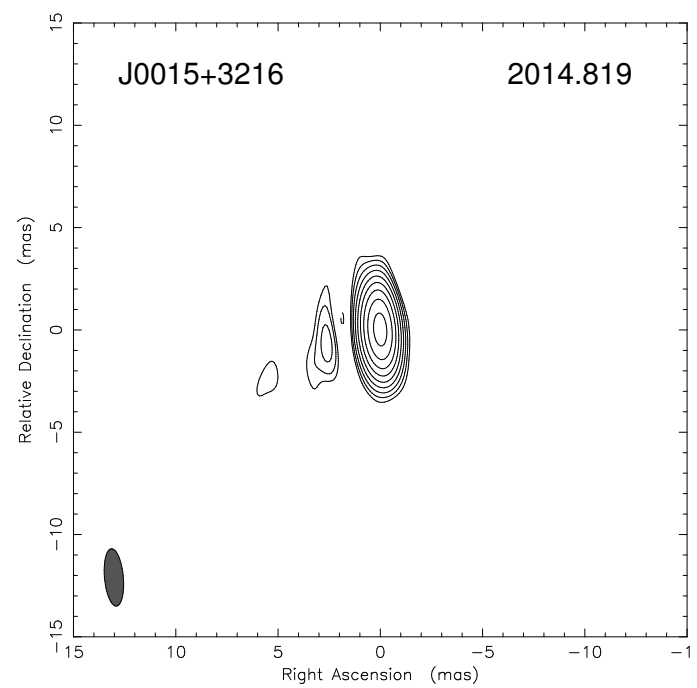

Fig. 16. EVN $5 \mathrm{GHz}$ image of J0015+3216 (calibrator of PW And) taken on 2014.819. The lowest contour level corresponds to 3 times the statistical rms noise $\left(0.3 \mathrm{mJy}\right.$ beam $\left.^{-1}\right)$ with a scale factor between contiguous contours of $\sqrt{3}$. The peak flux density in the image is $0.21 \mathrm{mJy} /$ beam.

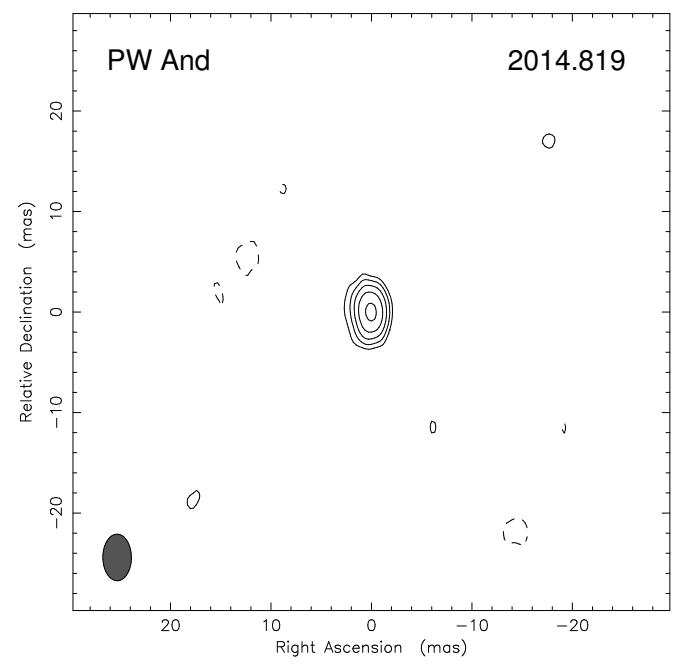

Fig. 17. EVN $5 \mathrm{GHz}$ image of PW And taken on 2014.819. The lowest contour level corresponds to 3 times the statistical rms noise $\left(0.01 \mathrm{mJy} \mathrm{beam}^{-1}\right)$ with a scale factor between contiguous contours of $\sqrt{2}$. The peak flux density in the image is $0.16 \mathrm{mJy}$ beam $^{-1}$. The restoring beam (shown in the bottom-left corner of the map) is an elliptical Gaussian of $4.63 \times 2.84$ mas (PA 0.40 ). 


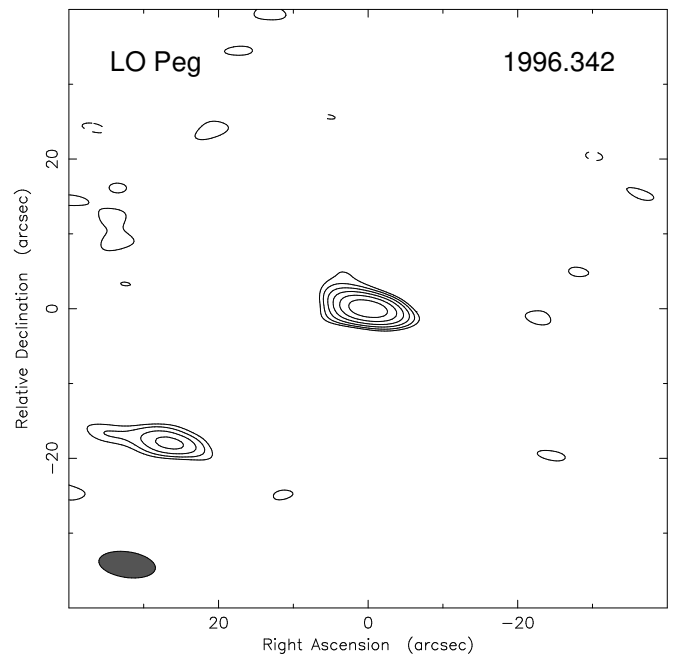

Fig. 18. VLA $8.4 \mathrm{GHz}$ image of LOPeg taken on 1996.342. The lowest contour level corresponds to twice the statistical rms noise $\left(0.02 \mathrm{mJy} \mathrm{beam}^{-1}\right)$ with a scale factor between contiguous contours of $\sqrt{2}$. The peak flux density in the image is $0.42 \mathrm{mJy} / \mathrm{beam}$. The restoring beam (shown in the bottom-left corner of the map) is an elliptical Gaussian of $7.63 \times 3.44 \operatorname{arcsec}(\mathrm{PA} 82: 0)$.

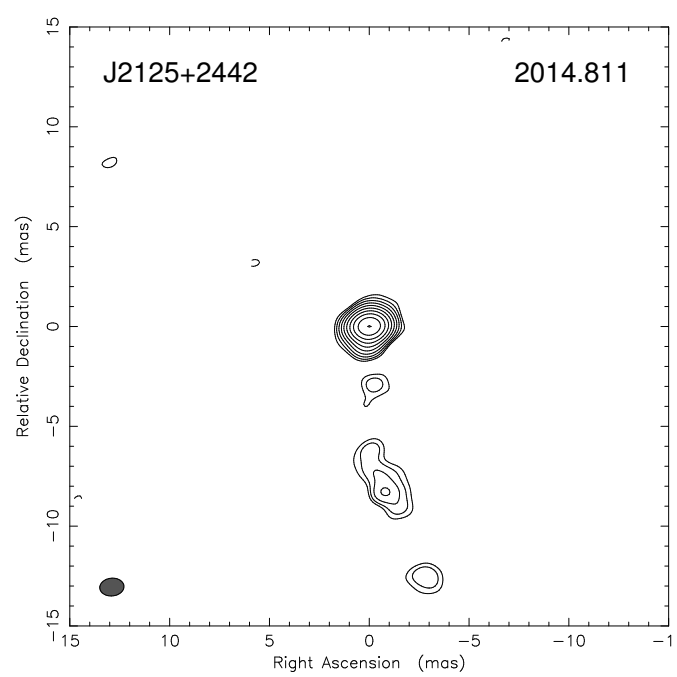

Fig. 19. EVN $5 \mathrm{GHz}$ image of J2125+2442 (calibrator of LO Peg) taken on 2014.811. The lowest contour level corresponds to 3 times the statistical $\mathrm{rms}$ noise $\left(0.2 \mathrm{mJy}\right.$ beam $\left.^{-1}\right)$ with a scale factor between contiguous contours of $\sqrt{3}$. The peak flux density in the image is $0.11 \mathrm{mJy} / \mathrm{beam}$.

detection of compact radio emission of the low-mass companion c (Azulay et al. 2014) allowed us to sample not only the absolute orbit of component A (with respect to the external quasar J1746+6226), but also the relative orbit, both of which are necessary to determine model-independent dynamical masses of the components of this system. The proximity of the two stars near periastron ( 20-40 mas in the last four years) has prevented an appropriate sampling of the relative orbit until the recent use of more precise interferometric techniques: aperture-masking (Evans et al. 2012) and VLBI (this work). The results of our orbital analysis yields values of $0.70 \pm 0.07 M_{\odot}$ and $0.45 \pm 0.04 M_{\odot}$ for components A and c, respectively, which are larger than the theoretical values predicted by PMS evolutionary tracks. The amount of this disagreement is $\sim 10 \%$ for component $\mathrm{A}$ and $10-40 \%$ for component c, contributing to the increasing observational evidence that PMS models underpredict the masses of systems with masses below $1.2 M_{\odot}$. We have found that the inclusion of the effect of the stellar magnetic field in the theoretical models tends to reduce such a discrepancy. Remarkably, our study allowed us to obtain a revised and more precise value of the parallax (31.4 \pm 0.5 mas $)$, thus solving a long-standing discussion about the distance to this system.

With respect to the other stars included in our study, EK Dra and PW And showed compact radio emission at milliarcsecond scales, meanwhile LO Peg, appeared to be "off" at the time of observations. Complementary, companion infrared observations of EK Dra permitted us a revision of the orbital parameters of this system.

Acknowledgements. R.A., J.C.G., J.M.M., and E.R. were partially supported by the Spanish MINECO projects AYA2012-38491-C02-01 and AYA201563939-C2-2-P and by the Generalitat Valenciana project PROMETEO/2009/104 and PROMETEOII/2014/057. R.A. acknowledges the Max-Planck-Institute für Radioastronomie for its hospitality. E.T. was supported by the "PRA 2016 Università di Pisa". E.T. also acknowledges the INFN iniziativa specifica TAsP.

\section{References}

Azulay, R., Guirado, J. C., Marcaide, J. M., Martí-Vidal, I., \& Arroyo-Torres, B. 2014, A\&A, 561, A38

Azulay, R., Guirado, J. C., Marcaide, J. M., et al. 2015, A\&A, 578, A16 Baraffe, I., Chabrier, G., Allard, F., \& Hauschildt, P. H. 1998, A\&A, 337, 403

Baraffe, I., Vorobyov, E., \& Chabrier, G. 2012, ApJ, 756, 118

Baraffe, I., Homeier, D., Allard, F., \& Chabrier, G. 2015, A\&A, 577, A42

Barnes, J. R., Collier Cameron, A., Lister, T. A., Pointer, G. R., \& Still, M. D. 2005, MNRAS, 356, 1501

Bell, C. P. M., Mamajek, E. E., \& Naylor, T. 2015, MNRAS, 454, 593

Bressan, A., Marigo, P., Girardi, L., et al. 2012, MNRAS, 427, 127

Chabrier, G., Baraffe, I., Allard, F., \& Hauschildt, P. 2000, ApJ, 542, 464

Chabrier, G., Gallardo, J., \& Baraffe, I. 2007, A\&A, 472, L17

Choi, J., Dotter, A., Conroy, C., et al. 2016, ApJ, 823, 102

Dorren, J. D., \& Guinan, E. F. 1994, ApJ, 428, 805

Duquennoy, A., \& Mayor, M. 1991, A\&A, 248, 485

Evans, T. M., Ireland, M. J., Kraus, A. L., et al. 2012, ApJ, 744, 120

Elliott, P., Bayo, A., Melo, C. H. F., et al. 2016, A\&A, 590, A13

Feiden, G. A. 2016, A\&A, 593, A99

Feiden, G. A., \& Chaboyer, B. 2012, ApJ, 757, 42

Feiden, G. A., \& Chaboyer, B. 2013, ApJ, 779, 183

Feiden, G. A., \& Chaboyer, B. 2014, ApJ, 789, 53

Fruchter, A. S., \& Hook, R. N. 2002, PASP, 114, 144

Gagné, J., Lafrenière, D., Doyon, R., Malo, L., \& Artigau, É. 2014, ApJ, 783, 121

Gálvez, M. C., Montes, D., Fernández-Figueroa, M. J., \& López-Santiago, J. 2006, Ap\&SS, 304, 59

Gennaro, M., Prada Moroni, P. G., \& Tognelli, E. 2012, MNRAS, 420, 986

Griffin, R. F. 2013, The Observatory, 133, 322

Griffin, R. F. 1992, The Observatory, 112, 41

Griffin, R. F., \& Filiz Ak, N. 2010, Ap\&SS, 330, 47

Güdel, M., Schmitt, J. H. M. M., Benz, A. O., \& Elias, N. M., II 1995, A\&A, 301,201

Guirado, J. C., Martí-Vidal, I., Marcaide, J. M., et al. 2006, A\&A, 446, 733

Guirado, J. C., Marcaide, J. M., Martí-Vidal, I., et al. 2011, A\&A, 533, A106

Hartigan, P., Strom, K. M., \& Strom, S. E. 1994, ApJ, 427, 961

Henry, G. W., Fekel, F. C., \& Hall, D. S. 1995, AJ, 110, 2926

Herczeg, G. J., \& Hillenbrand, L. A. 2014, ApJ, 786, 97

Hillenbrand, L. A., \& White, R. J. 2004, ApJ, 604, 741

Hormuth, F., Brandner, W., Hippler, S., Janson, M., \& Henning, T. 2007, A\&A, 463, 707

Hormuth, F., Hippler, S., Brandner, W., Wagner, K., \& Henning, T. 2008, The International Society for Optical Engineering, 7014, 701448

Janson, M., Brandner, W., Lenzen, R., et al. 2007, A\&A, 462, 615

Järvinen, S. P., Berdyugina, S. V., \& Strassmeier, K. G. 2005, A\&A, 440, 735

Jeffries, R. D., Byrne, P. B., Doyle, J. G., et al. 1994, MNRAS, 270, 153

Kastner, J. H., Rapson, V., Sargent, B., Smith, C. T., \& Rayner, J. 2015, 18th

Cambridge Workshop on Cool Stars, Stellar Systems, and the Sun, 18, 313

König, B., Guenther, E. W., Woitas, J., \& Hatzes, A. P. 2005, A\&A, 435, 215

Lafrenière, D., Doyon, R., Marois, C., et al. 2007, ApJ, 670, 1367

Lim, J. 1993, ApJ, 405, L33

Lim, J., White, S. M., Nelson, G. J., \& Benz, A. O. 1994, ApJ, 430, 332 
R. Azulay et al.: Young, active radio stars in the AB Doradus moving group

López-Santiago, J., Montes, D., Fernández-Figueroa, M. J., \& Ramsey, L. W. 2003, A\&A, 411, 489

López-Santiago, J., Montes, D., Crespo-Chacón, I., \& Fernández-Figueroa, M. J. 2006, ApJ, 643, 1160

Lowrance, P. J., Becklin, E. E., Schneider, G., et al. 2005, AJ, 130, 1845

Luhman, K. L., Stauffer, J. R., Muench, A. A., et al. 2003, ApJ, 593, 1093

Malo, L., Doyon, R., Lafrenière, D., et al. 2013, ApJ, 762, 88

Mathieu, R. D., Baraffe, I., Simon, M., Stassun, K. G., \& White, R. 2007, Protostars and Planets V, 411

McCarthy, K., \& White, R. J. 2012, AJ, 143, 134

Metchev, S. A., \& Hillenbrand, L. A. 2004, ApJ, 617, 1330

Montes, D., López-Santiago, J., Fernández-Figueroa, M. J., \& Gálvez, M. C. 2001, A\&A, 379, 976

Pandey, J. C., Singh, K. P., Drake, S. A., \& Sagar, R. 2005, AJ, 130, 123

Pecaut, M. J., \& Mamajek, E. E. 2013, ApJS, 208, 9

Pecaut, M. J. 2016, Young Stars \& Planets Near the Sun, 314, 85

Piluso, N., Lanza, A. F., Pagano, I., Lanzafame, A. C., \& Donati, J.-F. 2008, MNRAS, 387, 237
Schlieder, J. E., Lépine, S., \& Simon, M. 2012, AJ, 143, 80

Shepherd, M. C., Pearson, T. J., \& Taylor, G. B. 1994, BAAS, 26 , 987

Siess, L., Dufour, E., \& Forestini, M. 2000, A\&A, 358, 593

Somers, G., \& Pinsonneault, M. H. 2015, ApJ, 807, 174

Stassun, K. G., Mathieu, R. D., Vaz, L. P. R., Stroud, N., \& Vrba, F. J. 2004, ApJS, 151, 357

Strassmeier, K. G., Hall, D. S., Zeilik, M., et al. 1988, A\&AS, 72, 291

Tognelli, E., Prada Moroni, P. G., \& Degl'Innocenti, S. 2011, A\&A, 533, A109

Tognelli, E., Degl'Innocenti, S., \& Prada Moroni, P. G. 2012, A\&A, 548, A41

Tognelli, E., Prada Moroni, P. G., \& Degl'Innocenti, S. 2015, MNRAS, 454, 4037

Torres, C. A. O., Quast, G. R., Melo, C. H. F., \& Sterzik, M. F. 2008, Handbook of Star Forming Regions, Volume II, 757

van Leeuwen, F. 2007, A\&A, 474, 653

Wichmann, R., Schmitt, J. H. M. M., \& Hubrig, S. 2003, A\&A, 399, 983

Zuckerman, B., \& Song, I. 2004, ARA\&A, 42, 685

Zuckerman, B., Song, I., \& Bessell, M. S. 2004, ApJ, 613, L65 